\title{
Identifying young stellar objects in nine Large Magellanic Cloud star-forming regions ${ }^{\star}$
}

\author{
L. R. Carlson ${ }^{1}$, M. Sewiło ${ }^{2}$, M. Meixner ${ }^{3}$, K. A. Romita ${ }^{4}$, and B. Lawton ${ }^{3}$ \\ 1 Leiden Observatory, Leiden, The Netherlands \\ e-mail: carlson@strw.leidenuniv.nl \\ 2 Johns Hopkins University, Baltimore, 3400 N. Charles St., Baltimore, MD, USA \\ 3 Space Telescope Science Institute, 3700 San Martin Drive, Baltimore, MD, USA \\ ${ }^{4}$ University of Florida, Gainesville, FL, USA
}

Received 12 December 2011 / Accepted 13 March 2012

\begin{abstract}
We introduce a new set of selection criteria for the identification of infrared bright young stellar object (YSO) candidates and apply them to nine HII regions in the Large Magellanic Cloud (LMC), focusing particularly on lower mass candidates missed by most surveys. Data are from the Spitzer Space Telescope legacy program SAGE (Surveying the Agents of Galaxy Evolution; Meixner et al. 2006, AJ, 132, 2268), combined with optical photometry from the Magellanic Clouds Photometric Survey (MCPS; Zaritsky et al. 1997, AJ, 114, 1002) and near-infrared photometry from the InfraRed Survey Facility (IRSF; Kato et al. 2007, PASJ, 59, 615). We choose regions of diverse physical size, star formation rates (SFRs), and ages. We also cover a wide range of locations and surrounding environments in the LMC. These active star-forming regions are LHA 120-N 11, N 44, N 51, N 105, N 113, N 120, N 144, N 160, and N 206. Some have been well-studied (e.g., N11, N44, N160) in the past, while others (e.g., N51, N144) have received little attention. We identify 1045 YSO candidates, including 918 never before identified and 127 matching previous candidate lists. We characterize the evolutionary stage and physical properties of each candidate using the spectral energy distribution (SED) fitter of Robitaille et al. (2007, ApJS, 169, 328) and estimate mass functions and SFRs for each region.
\end{abstract}

Key words. stars: formation - stars: protostars - HII regions - photon-dominated region (PDR) - galaxies: star clusters: general Magellanic Clouds

\section{Introduction}

The nearby Large Magellanic Cloud (LMC) is an excellent laboratory for the study of the life-cycle of matter from relatively quiescent interstellar medium (ISM) through star formation, main sequence (MS) stellar existence, and eventual feedback of material as stars evolve and die. It is well situated for observations, far from the confusion and the extinction of the Galactic plane and near enough for high-resolution observations. At a distance of $\sim 50 \mathrm{kpc}$ (e.g., Panagia 2005; Schaefer 2008), the resolution is $\sim 14.5 \mathrm{pc} / \mathrm{arcmin}$. The metallicity falls within the range $Z=0.3-0.5 Z_{\odot}$ (cf. Dufour et al. 1982; Westerlund 1997), similar to that of galaxies at redshift $z \sim 1.5$ (Pei et al. 1999), a time of peak galaxy formation. Morphologically, the LMC has an evident bar structure and a disk component, seen from a relatively face-on viewing-angle $\left(\sim 24-35^{\circ}\right.$; Weinberg \& Nikolaev 2001; van der Marel \& Cioni 2001) such that the distance to each source is effectively identical.

Lists of young stellar object (YSO) candidates covering the entire $7^{\circ} \times 7^{\circ}$ area imaged by the Spitzer Space Telescope (Spitzer) legacy program "Surveying the Agents of Galaxy Evolution" (SAGE; Meixner et al. 2006) have been created by Whitney et al. (2008) and Gruendl \& Chu (2009), hereafter W08

* Full Tables 1-3, 5 are only available at the CDS via anonymous ftp to cdsarc.u-strasbg. fr $(130.79 .128 .5)$ or via

http://cdsarc.u-strasbg.fr/viz-bin/qcat?J/A+A/542/A66 and GC09, using different strategies. These initial surveys focus on the most massive and embedded YSOs which are the easiest to identify and suffer the least contamination in terms of color selection. The study of W08 employs a set of selection criteria which are simple in philosophy but complex in implementation. They use several different color-magnitude diagrams (CMDs) to select as many YSO candidates as possible while minimizing the selection of background galaxies and evolved stars. Selection is guided by YSO-model color-magnitude space contrasted with that of observed "galaxies" and evolved star models. They then rely upon spectral energy distribution (SED) fitting to determine the likelihood that a given source is a YSO and remove some galaxies identified by eye in Spitzer InfraRed Array Camera (IRAC; Fazio et al. 2004) or $24 \mu \mathrm{m}$ Multiwavelength Imaging Photometer for Spitzer (MIPS; Rieke et al. 2004) images. This approach is rigorous and uniform but conservative due to the use of the strictly-defined SAGE Epoch 1 Point Source Catalog (Meixner et al. 2006) ${ }^{1}$ of point spread function (PSF) fit sources with high signal-to-noise ratios and the use of conservative color-magnitude cuts. GC09 use one, simple color selection (which excludes candidates less massive than $\sim 4 M_{\odot}$ and all sources without measured flux in either 4.5 or $8.0 \mu \mathrm{m}$ ) and their own less-stringent catalog of aperture photometry. From the

\footnotetext{
SAGE data and documentation are available from http://irsa.ipac. caltech. edu/data/SPITZER/SAGE/
} 
color-selected list, they then identify probable YSOs via visual inspection of SEDs and images. The two catalogs complement one another, with the $\sim 1000$ W08 sources including many fainter sources that do not fall within the GC09 color-selection, and the 1172 GC09 candidates including more extended sources and sources in more complicated environments. Both of these initial surveys are, however, incomplete in at least two ways. They miss less embedded massive YSOs (e.g., Stage II and Stage III) and low luminosity (lower mass) YSOs.

We present a streamlined set of YSO candidate selection criteria, using five color-magnitude combinations which include all four IRAC bands and MIPS $24 \mu \mathrm{m}$. These data are described in Sect. 2. After initial color selection (Sect. 3.1), we fit SEDs to estimate physical parameters, using the model grid and fitter of Robitaille et al. (2006, 2007). We require better fits for sources fulfilling fewer color criteria, as described in Sect. 3.2. We apply our selection criteria to optical through Mid-infrared (Mid-IR) photometry for nine HII regions in the LMC: LHA 120-N 11, LHA 120-N 44, LHA 120-N 51, LHA $120-\mathrm{N} \quad 105$, LHA 120-N 112, LHA 120-N 120, LHA 120-N 144, LHA 120-N 160, and LHA 120-N 206 (hereafter N11, etc.; Henize 1956), detailed in Sect. 4. We show the efficacy of our new YSO candidate selection scheme, provide a comparison to other YSO candidate lists, and examine discrepancies in Sect. 5.1. In Sect. 5.2, we provide star-formation rate (SFR) estimates for each region. Section 6 encompasses some final remarks.

\section{Data}

Our data are from SAGE in the Spitzer/IRAC 3.6, 4.5, 5.8, and $8.0 \mu \mathrm{m}$ bands and MIPS $24 \mu \mathrm{m}$ which we use for color-selection. We then fit the SEDs to select the strongest YSO candidates. We add photometry in the near-IR bands $J, H$, and $K_{\mathrm{s}}$ from the Magellanic Clouds Point Source Catalog created with the Simultaneous 3-color IR Imager for Unbiased Surveys (SIRIUS) aboard the InfraRed Survey Facility (IRSF) by Kato et al. (2007). We convert these to the Two-Micron All Sky Survey (2MASS; Skrutskie et al. 2006) photometric system for compatibility with the SED fitter, following the discussion in Kato et al. (2007). We use optical photometry from the Magellanic Clouds Photometric Survey (MCPS; Zaritsky et al. 1997) in the $U, B, V$, and $I$ bands. SAGE data processing is described by Meixner et al. (2006), and public catalogs are available through the Spitzer Science Center ${ }^{2}$. The IRAC angular resolution is $\sim 2 "$. Photometric completeness is expected to be $>99 \%$ down to $16,16,14$, and $13.5 \mathrm{mag}$ in [3.6], [4.5], [5.8], and [8.0], respectively, and 50\% down to $18,17,15.5$, and $15 \mathrm{mag}$. We provide photometry for all YSO candidates in our 9 regions in Tables 1 and 2, with mosaicked IRAC photometry matched to MCPS, IRSF, and MIPS $24 \mu \mathrm{m}$ within $1^{\prime \prime}$.

\section{Identification of YSOs}

We present a simplified procedure (compared to that of W08) for the color-selection of YSO candidates. We use only five infrared color-magnitude combinations, making sure to use Spitzer/IRAC 3.6, 4.5, 5.8, and $8.0 \mu \mathrm{m}$ bands and MIPS $24 \mu \mathrm{m}$. In each CMD, we select an area in which there is a high density of model YSOs (from Robitaille et al. 2006) and a low density

\footnotetext{
2 We use the list "Single Frame + Mosaic Photometry Archive" as described in the documentation on http://irsa.ipac. caltech.edu/ data/SPITZER/SAGE/
}

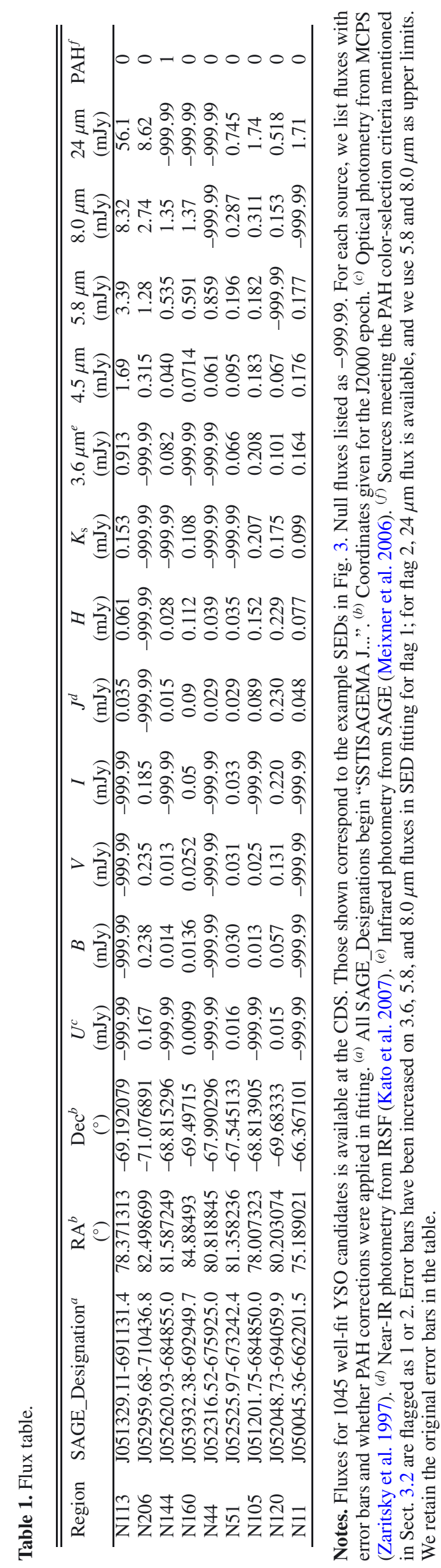



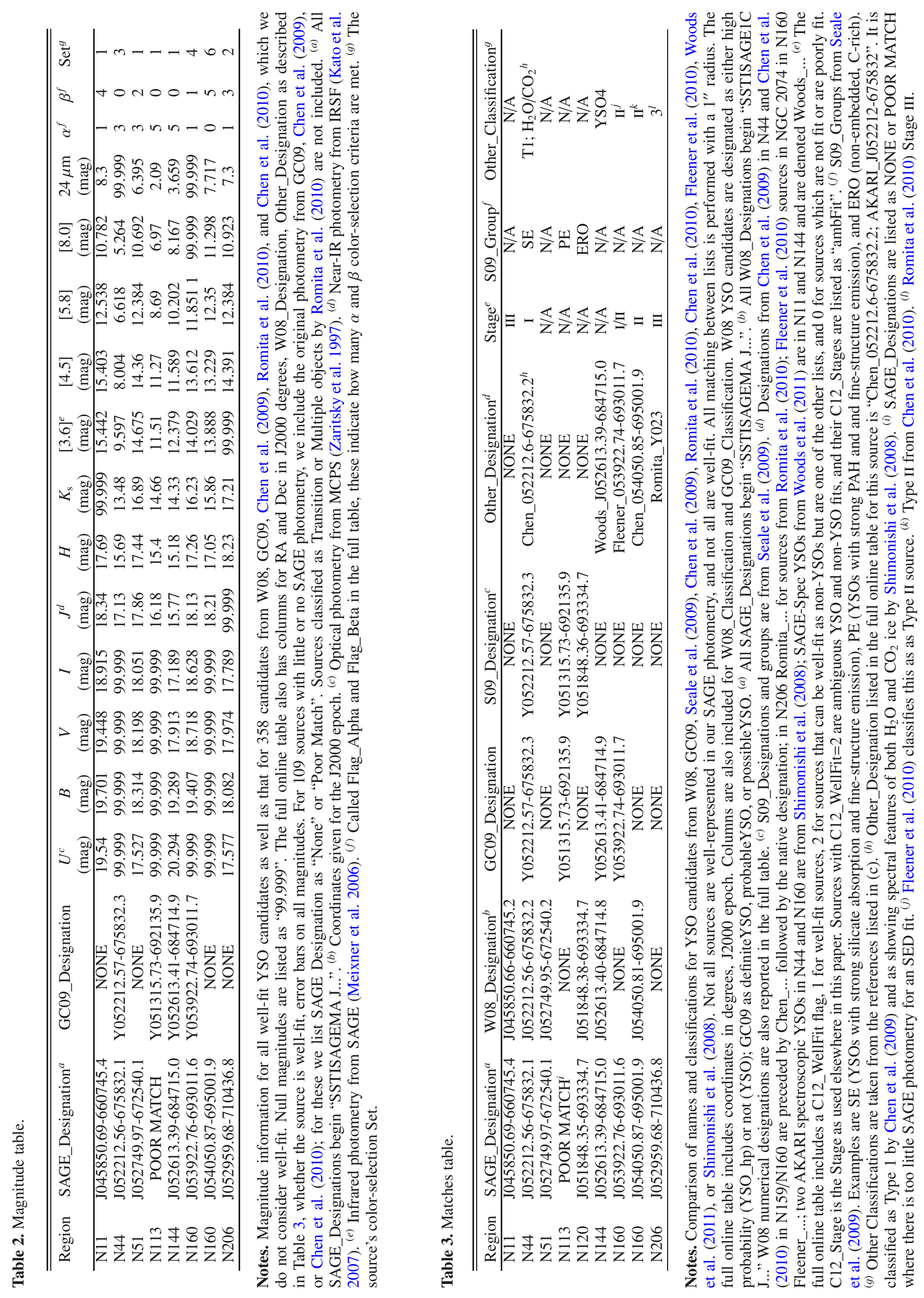
Table 4. LMC "well-fit" YSO candidate fitting requirements.

\begin{tabular}{lccccc}
\hline \hline Set & $\chi_{\min }^{2} / p t$ & $\alpha$ & $\beta$ & $\alpha+\beta$ & Bands \\
\hline Set 1 & $<15$ & $\geq 1$ & - & 5 & 5 \\
Set 2 & $<12$ & $\geq 1$ & - & 4 & 4 \\
Set 3 & $<10$ & $\geq 1$ & - & 3 & 4 \\
Set 4 & $<8$ & $\geq 1$ & - & 2 & 4 \\
Set 5 & $<6$ & 1 & 0 & 1 & 4 \\
\hline Set 6 & $<5$ & 0 & 5 & 5 & 5 \\
Set 7 & $<3.5$ & 0 & 4 & 4 & 5 \\
Set 8 & $<2.5$ & 0 & 3 & 3 & 5 \\
Set 9 & $<1.5$ & 0 & 2 & 2 & 5 \\
\hline
\end{tabular}

of other types of sources; these are our $\alpha$ color selections and are described in detail in Sewiło et al. (in prep.). A secondary $(\beta)$ set of criteria complement the $\alpha$ selections, sampling CMD space with more "contamination" from galaxies and evolved stars but a high density of YSO models, particularly those of low mass. With the high degree of contamination, we consider $\beta$ criteria appropriate only for known star-forming regions with high background emission, where background galaxies are largely blocked by the ISM, and YSOs are more abundant than asymptotic giant branch stars (AGBs). We apply the SED fitter of Robitaille et al. (2007) to the color-selected sources and select our final list of "well-fit" YSO candidates according to the number of color-criteria met and the quantitative goodness $\left(\chi^{2} / \mathrm{pt}\right)$ of the best fits.

\subsection{Color selection}

Our YSO candidate color selections are plotted in Figs. 1 and 2 and described by Eqs. (3)-(10). The $\alpha$ criteria are appropriate for galaxy-wide surveys; we expect very low contamination from non-YSO sources. We designed these $\alpha$ criteria for the Small Magellanic Cloud (Sewiło et al., in prep.) and scale here for the LMC distance. These yield YSO candidate lists similar to those of the other surveys, identifying only luminous, high-mass YSO candidates. The $\beta$ set of criteria is to be used in star-forming regions where color-selected sources are significantly more likely to be YSOs than background galaxies or evolved stars, choosing color-magnitude space in which we still expect most sources to be YSOs but in which (galaxy-wide) there is significant contamination from non-YSO sources. Our $\beta$-selections are appropriate only in these dusty, star-forming regions in which YSO candidates are concentrated along dust lanes, where we can reasonably expect only a small percentage of contaminants.

Figures 1 and 2 shows the five CMDs with $\alpha$ and $\beta$ selection criteria indicated. The full list of LMC photometric sources is shown in a density plot in the background in Fig. 1, and the Robitaille et al. (2006) YSO models are the background in Fig. 2. Our selections avoid the primary "vertical" population of MS and giant stars. YSO models from Robitaille et al. (2006) have little overlap with this "vertical" population. YSO models do overlap significantly with the color-magnitude locations of evolved sources (candidate AGB stars from Srinivasan et al. 2009, are marked in Fig. 1) and galaxy candidates (here "blank" fields from the edges of the SAGE images, as in W08, also in Fig. 1). We mark previously identified LMC YSO candidates ("List YSO Candidates" from W08 and GC09) for the full LMC in Fig. 1 and for the examined regions only in Fig. 2, noting their distinct CMD locations, the result of using different color-criteria. Our final list of Well-Fit YSO Candidates from these nine star-forming regions is also plotted in Fig. 2. Planetary nebulae from the Reid \& Parker (2006) $\mathrm{H} \alpha$ study, identified by Hora et al. (2008) in the SAGE data, do contaminate the full color-magnitude space and are unavoidable in IR colorselection. However, like AGB stars, planetary nebulae are not expected to be abundant in young star formation regions.

$$
\alpha_{3658}\left\{\begin{array}{rrrrr}
{[3.6] \leq 13.2} & \text { and } & ([3.6]-[5.8])>2.1 \\
\text { or } & 10.5<[3.6] \leq 13.2 & \text { and } & 0.8<([3.6]-[5.8]) \leq 2.1 \\
\text { or } & {[3.6]>13.2} & \text { and } & ([3.6]-[5.8])>3.0
\end{array}\right\}
$$

$$
\beta_{3658}\left\{\begin{array}{rrrl} 
& {[3.6] \leq 8.0} & \text { and } & 0.8<([3.6]-[5.8]) \leq 2.1 \\
\text { or } & 8.0<[3.6] \leq 10.5 & \text { and } & 1.6<([3.6]-[5.8]) \leq 2.1 \\
\text { or } & {[3.6]>13.2} & \text { and } & 0.8 \leq([3.6]-[5.8]) \leq 3.0
\end{array}\right\}
$$

$$
\alpha_{4558}\left\{\begin{array}{rrrr} 
& {[4.5] \leq 12.5} & \text { and } & ([4.5]-[5.8])>1.1 \\
\text { or } & {[4.5] \leq 12.5} & \text { and } & 0.5<([4.5]-[5.8]) \leq 1.1 \\
& & \text { and } & (10.833-1.667 \times([4.5]-[5.8]))<[4.5] \\
\text { or } & {[4.5]>12.5} & \text { and } & (7.0+5.0 \times([4.5]-[5.8]))>[4.5]
\end{array}\right\}
$$

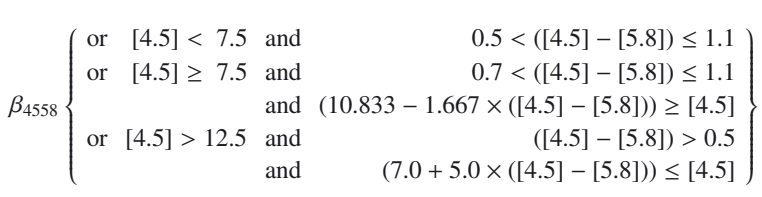

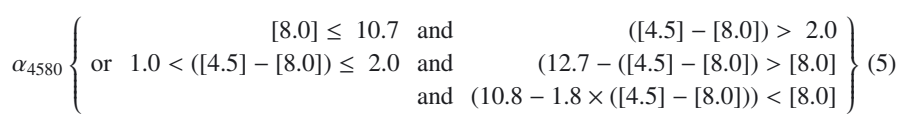

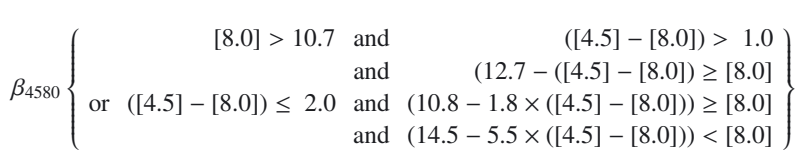

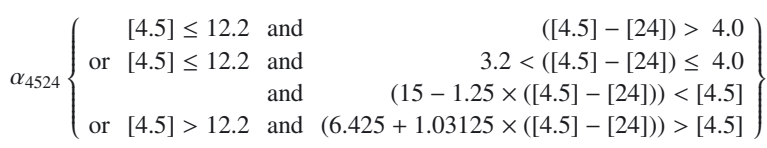

$$
\beta_{4524}\left\{\begin{array}{rrrr}
3.2<([4.5]-[24]) \leq 4.0 & \text { and } & (15-1.25 \times([4.5]-[24])) \leq[4.5] \\
\text { or } & {[4.5]>12.2} & \text { and } & (6.425+1.03125 \times([4.5]-[24])) \leq[4.5] \\
& & \text { and } & ([4.5]-[24])>3.2
\end{array}\right\}
$$

$$
\alpha_{8024}\left\{\begin{array}{rrr}
{[8.0] \leq 10.0 \text { and }} & ([8.0]-[24])>2.7 \\
\text { or }[8.0] \leq 10.0 \text { and } & \text { and } & (12-1.667 \times([8.0]-[24]) \leq 2.7 \\
& \text { and } & [24]))<[8.0] \\
\text { or }[8.0]>10.0 & \text { and } & (5.7195-1.0976 \times([8.0]-[24]))>[8.0]
\end{array}\right\}
$$

$\beta_{8024}\left\{\begin{array}{rrrr}1.5<([8.0]-[24])) \leq 2.7 & \text { and } & (10.501-1.667 \times([8.0]-[24])))<[8.0] \\ & & \text { and } & (12-1.667 \times([8.0]-[24])) \geq[8.0] \\ \text { or } & {[8.0]>10.0} & \text { and } & ([8.0]-[24]))>1.5 \\ & & \text { and } & (5.7195-1.0976 \times([8.0]-[24])) \leq[8.0]\end{array}\right\}$ (10)

\subsection{YSO candidate selection and classification}

We use the YSO model grid and fitter of Robitaille et al. (2006, 2007) to determine which sources are well-fit by YSO models. The grid incorporates 20000 model YSOs with a wide range of physical parameters, each with SEDs generated for 10 viewing 


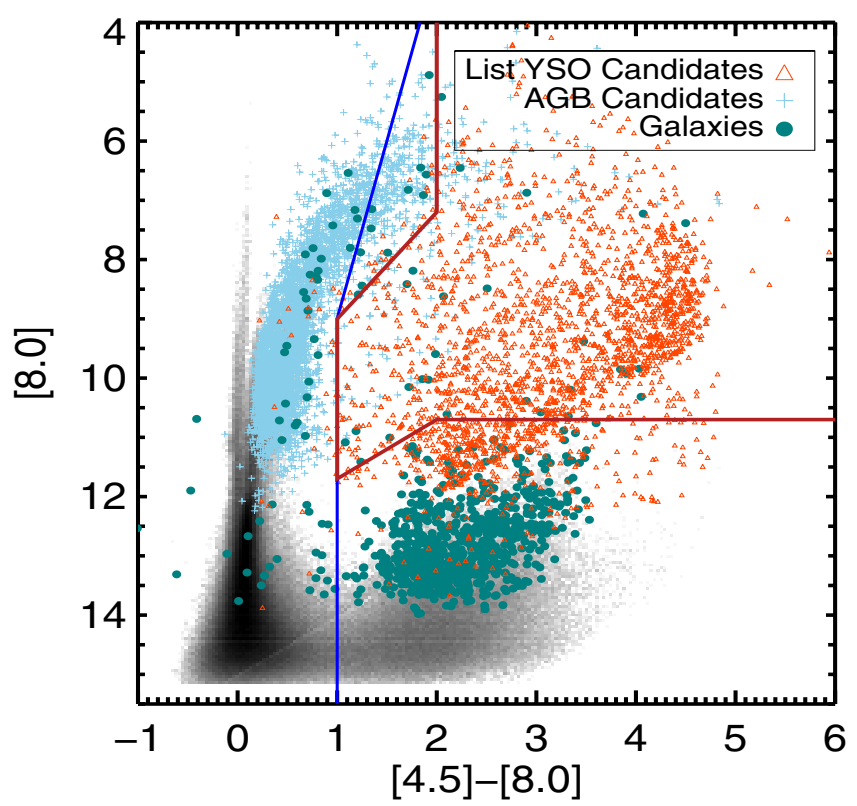

(a)

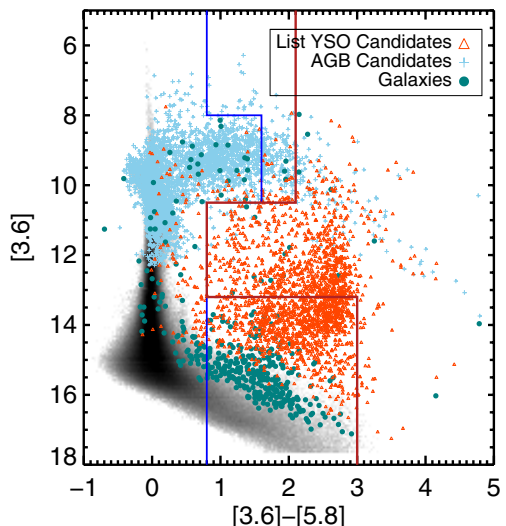

(b)

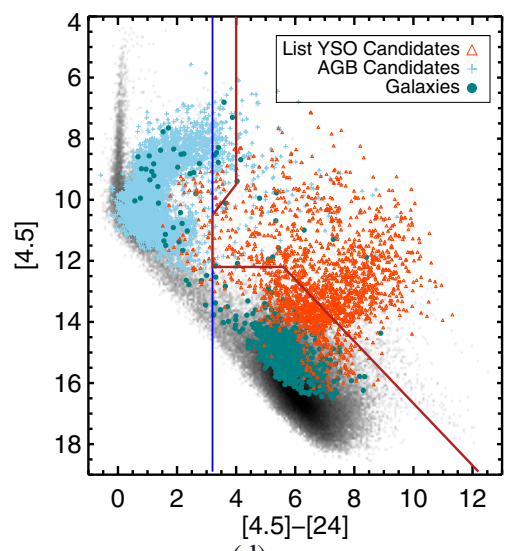

(d)

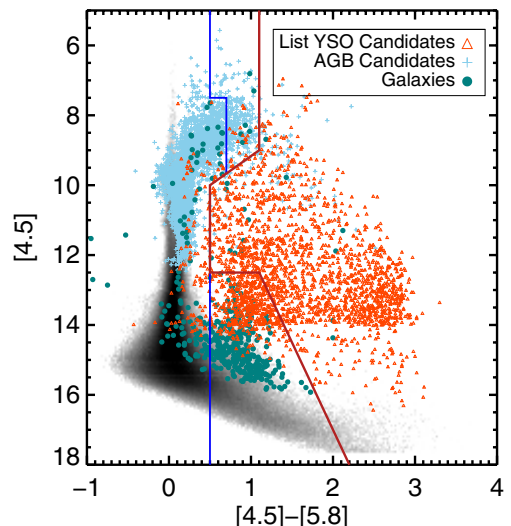

(c)

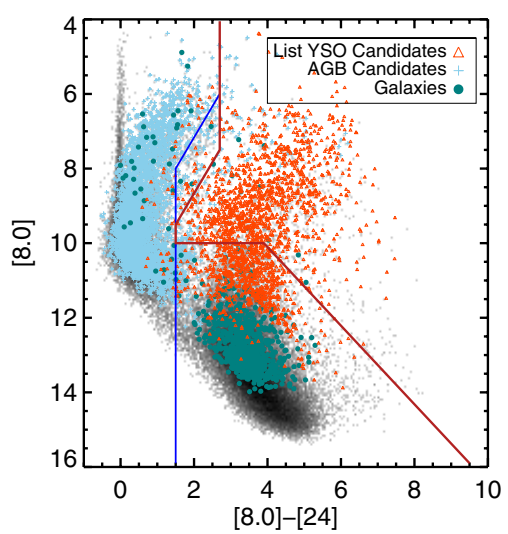

(e)

Fig. 1. CMDs showing $\alpha$ (red lines) and $\beta$ (blue lines) selection criteria on full LMC data. To the right (redward) of the red line is the $\alpha$ section of the CMD, where we expect to find massive YSOs and little contamination from non-YSO sources. The blue lines mark the boundary of the $\beta$-selection, in which we expect more contamination, particularly from background galaxies. List YSO candidates plotted are the full lists of W08 and GC09.

angles, for a total of 200000 SEDs. The fitter allows us to specify included photometric bands and applied apertures. The fitter calculates $\chi^{2}$ and $\chi^{2} / p t$ to estimate the goodness of fits, and for a source to be considered well-fit, we require $\chi_{\min }^{2} / \mathrm{pt}$ as listed in Table 4. We also discard sources best fit by AGB, galaxy, or MS templates. 


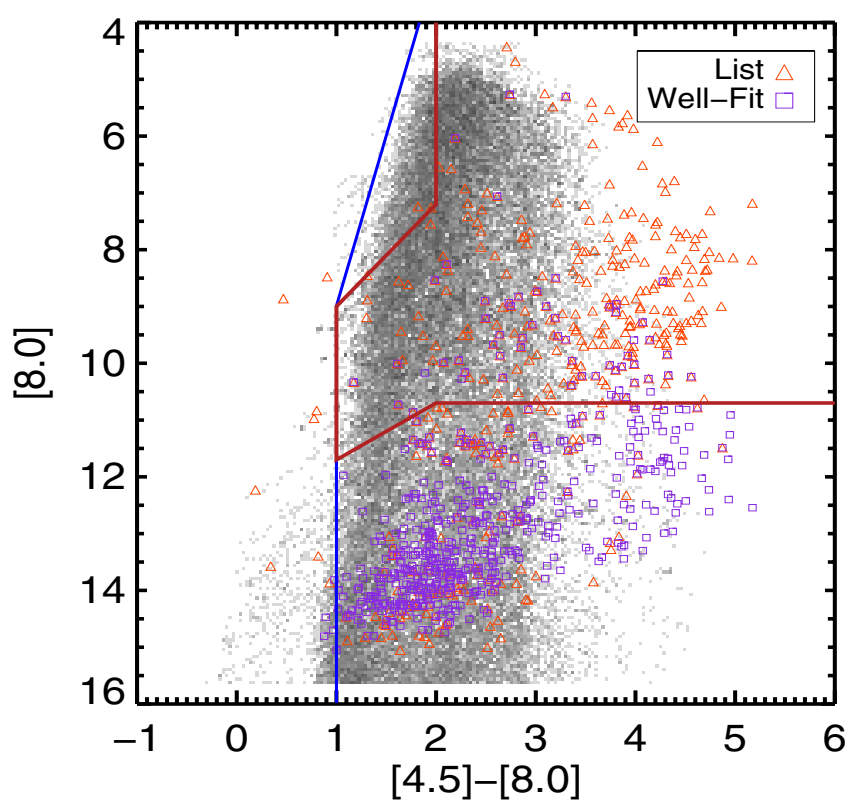

(a)

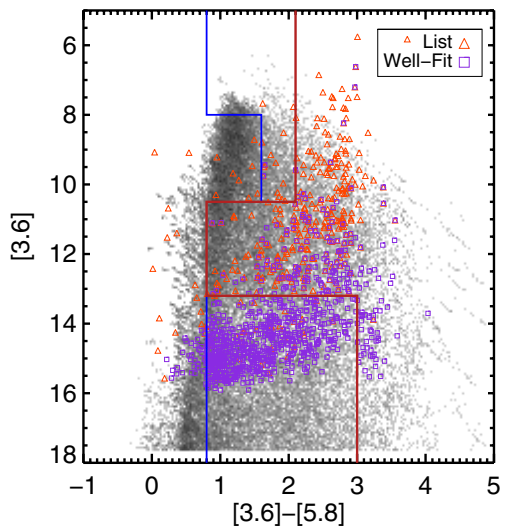

(b)

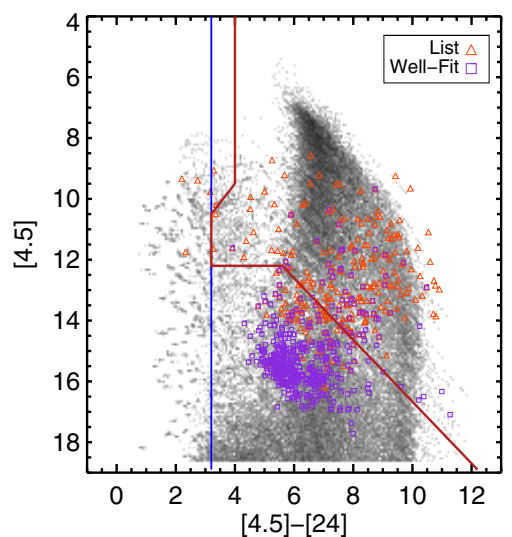

(d)

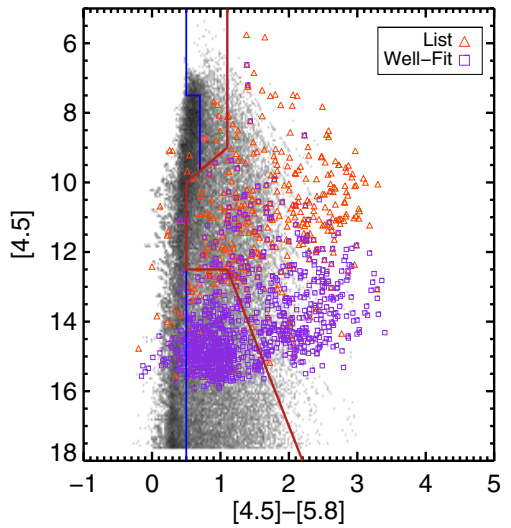

(c)

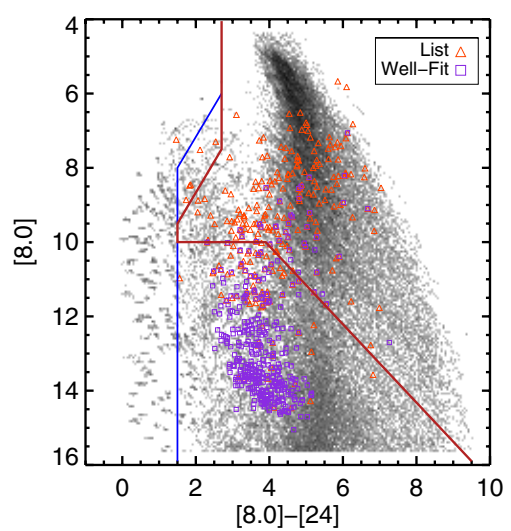

(e)

Fig. 2. CMDs showing $\alpha$ (red lines) and $\beta$ (blue lines) selection criteria with YSO candidates from these regions. The background is from the YSO models of Robitaille et al. (2006). List YSO candidates are from W08, GC09, Chen et al. (2009), Romita et al. (2010), and Chen et al. (2010) for these regions only. Well-fit YSO candidates are our final list of well-fit sources from this paper.

We apply the SED fitter to 9 Sets of sources, according to how many selection criteria are met. Requirements for each Set are shown in Table 4. Sources falling within at least one $\alpha$-selection area are considered quite reliable. The brightest, most massive sources are extended or saturated in multiple bands, and we do not expect them to appear in all of the 


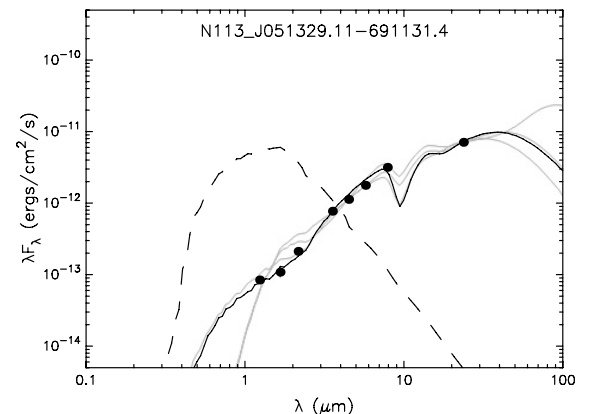

(a) Set 1

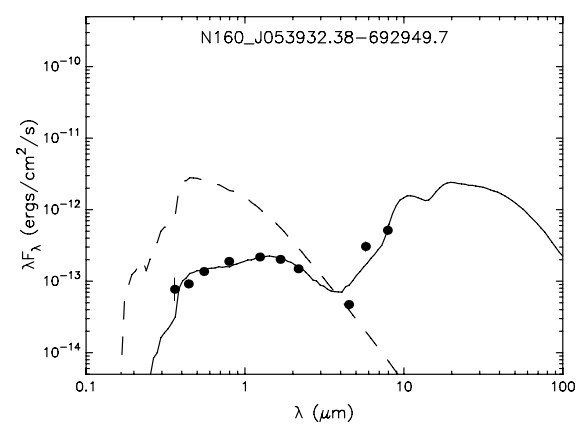

(d) Set 4

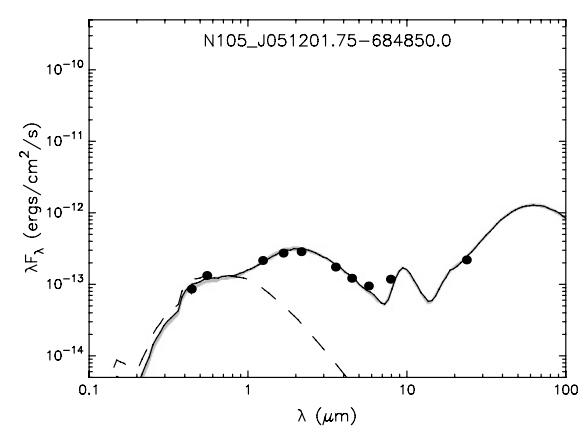

(g) Set 7

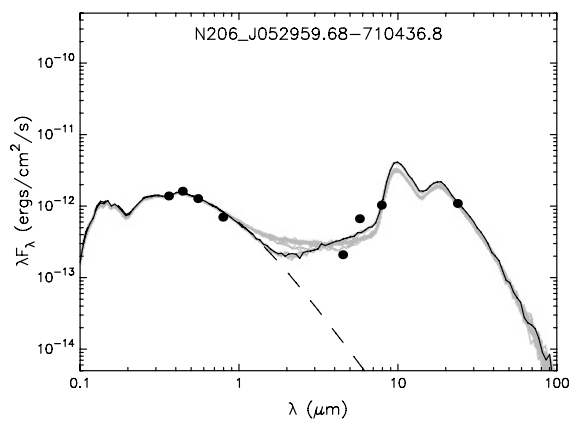

(b) Set 2

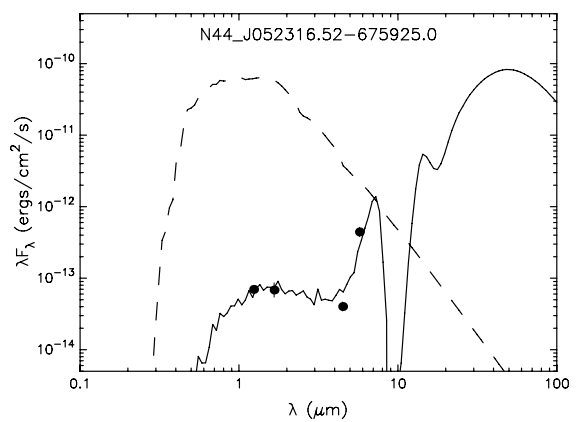

(e) Set 5

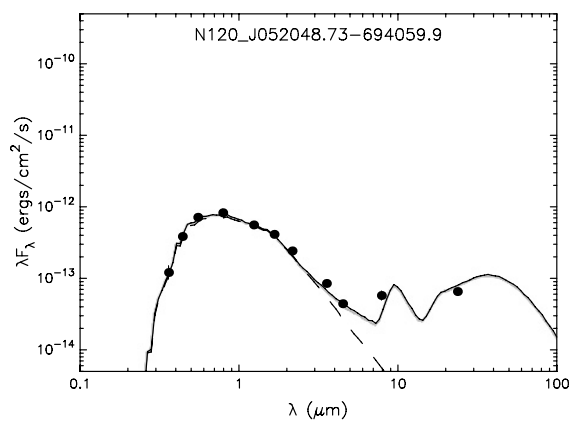

(h) Set 8

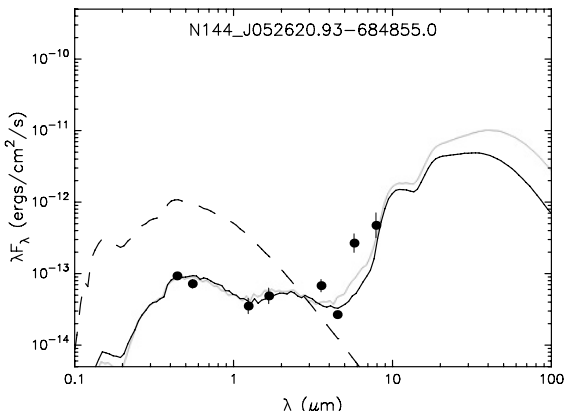

(c) Set 3

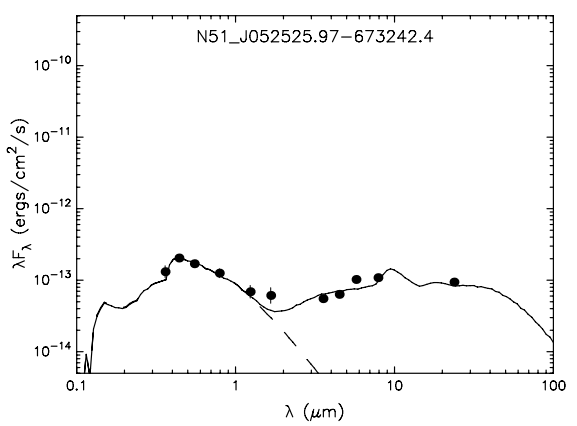

(f) Set 6

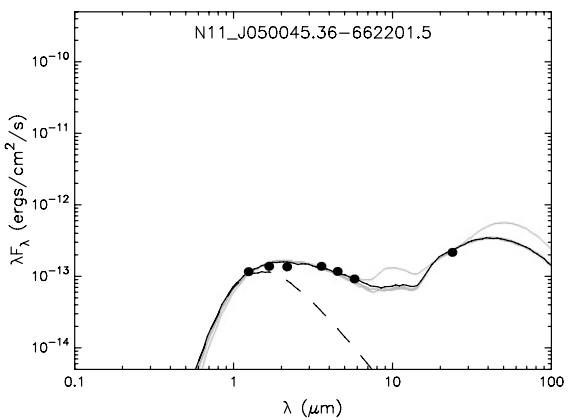

(i) Set 9

Fig. 3. Example SED for each selection Set detailed in Table 4. Error bars on fluxes are shown but usually less than the size of the dot. The best fit is shown in black with other fits with $\chi^{2} / p t \leq \chi_{\min }^{2} / p t+1$ shown in grey. The dashed line shows the photospheric emission from the central source. These are the same fits used to determine approximate physical and evolutionary properties. SEDs shown in a), c), d), and e) are classified as Stage I, i) as Stage I/II, f) and $\mathbf{h}$ ) as Stage II, and b) and g) as Stage III. The characteristic dip in SED c) at $4.5 \mu \mathrm{m}$, compared to the other three IRAC bands, indicates the presence of strong PAH emission, and error bars have been increased in 3.6, 5.8, and $8.0 \mu \mathrm{m}$, as described in the text.

selection CMDs. For fitting, we require sources meeting at least $1 \alpha$ criterion to have at least 4 bands of photometry defining the SED and consider a relatively large $\chi_{\min }^{2} / p t$ well-fit (i.e. reduced $\chi^{2}$; see Carlson et al. 2011, for a detailed explanation). We consider sources with at least $1 \alpha$ selection criterion and falling within either an $\alpha$ or $\beta$ selection region in all five CMDs to be the most reliable. Given the higher likelihood of contamination from non-YSO sources in our $\beta$ selections, we require better quality SED fits for $\beta$-selected sources. In order to be considered in our initial color-selected list, we also require that a source meets at least $2 \beta$ criteria if it meets no $\alpha$ criteria. For these, we require 5 bands of photometry for fitting and smaller $\chi_{\min }^{2} / p t$ values to be called well-fit. Example SEDs are shown in Fig. 3.

We use the calculated error flux bars in SED fitting, typically on the order of $10 \%$, but caveats should be noted. First, at the distance of the LMC all of our effective apertures are larger than the largest apertures calculated for the model YSOs from Robitaille et al. (2007). Also, the photometric apertures differ between the surveys, so each is encompassing a slightly different area. We match sources in the various catalogs to IRAC sources within a search radius of $1^{\prime \prime}$ and do expect a some false matches; visual inspection of automated matches indicates $\sim 2 \%$ contamination by false matches to IRAC. The conversion from IRSF to 2MASS magnitudes introduces an additional error, but this is estimated included in the conversion as given by Kato et al. (2007). Studies such as Carlson et al. (2011) and Chen et al. (2009) have shown that the large majority ( $70 \%$ or more) of IRAC YSO candidates at these distances are in fact (IR) dominant members of (optical) protoclusters. The YSO model set does not include multiple sources. However, we showed in Carlson et al. (2011) that the SED fitter can be used in the identification of YSOs even when they are members of protoclusters. This is in part because we expect one or two sources to dominate the luminosity of a protocluster: $L_{\mathrm{YSO}} \propto M_{\mathrm{YSO}}^{3}$ (i.e., Bernasconi \& Maeder 1996), though different sources may dominate in different wavelength regimes. Estimating how much 
each of these factors might contribute to the overall error budget for SED fitting is difficult. Our $\chi^{2} / p t$ values for sources to be considered well-fit (Table 4) were chosen empirically with a knowledge of the data and of these issues.

Polycyclic-aromatic hydrocarbons (PAHs), considered star formation indicators, can contribute significantly to the measured fluxes in the 3.6, 5.8, and $8.0 \mu \mathrm{m}$ bands but are not yet incorporated into the YSO models (Robitaille et al. 2006). We are able to estimate their potential contribution to SEDs. The [4.5] IRAC band is unaffected by PAHs, such that sources with significant PAH emission display a characteristic dip in their SEDs around $4.5 \mu \mathrm{m}$. In Carlson et al. (2011), we found that these sources fell within the color range $([3.6]-[4.5])<0.5$ and $1.5<([4.5]-[5.8])$. For sources meeting these color-criteria, we adjust our treatment of IRAC fluxes in SED fitting. Where no $24 \mu \mathrm{m}$ measurement is available, we increase flux error bars from the $\sim 10 \%$ calculated photometric errors to $20 \%, 30 \%$, and $40 \%$ in $3.6,5.8$, and $8.0 \mu \mathrm{m}$, respectively. Where a $24 \mu \mathrm{m}$ measurement is available, we increase the error bar on $3.6 \mu \mathrm{m}$ and set $5.8 \mu \mathrm{m}$ and $8.0 \mu \mathrm{m}$ fluxes as upper-limits. Notice that PAH contributions will move sources redward in and brighter in [8.0] v. [4.5]-[8.0] and in [3.6] v. [3.6]-[5.8] and redward in [4.5] v. [4.5]-[5.8]. We expect no effect on [4.5] v. [4.5]-[24]. Only in [8.0] v. [8.0]-[24] do we expect PAH sources to appear more blue. Indeed, many of our final well-fit and list YSO candidates lie redward of the color-magnitude area covered by the YSO models in Figs. 2a and c.

We classify our well-fit YSO candidates into the evolutionary Stages I-III defined by Robitaille et al. (2006), based on the fractional disk mass and the envelope accretion rate:

1. Stage I: embedded Source $\left(\dot{M}_{\text {env }}>10^{-6} M_{\star} \mathrm{yr}^{-1}\right)$;

2. Stage II: source with Disk $\left(\dot{M}_{\text {env }}<10^{-6} M_{\star} \mathrm{yr}^{-1}\right.$ and $\left.M_{\text {disk }} / M_{\star}>10^{-6}\right)$

3. Stage III: source with Optically Thin Disk or No Disk $\left(\dot{M}_{\mathrm{env}}<10^{-6} M_{\star} \mathrm{yr}^{-1}\right.$ and $\left.M_{\text {disk }} / M_{\star}<10^{-6}\right)$.

Stage I models are the least evolved, most embedded sources with significant ongoing accretion from their circumstellar envelopes. Stage II sources are surrounded by optically thick disks, and Stage III sources have optically thin disks or no disks. We list sources that can be well-fit by both Stage I and Stage II models as Stage I/II and sources fit by both Stage II and Stage III as Stage II/III. Some sources are well-fit by models covering all three stages; we consider these "Unclassified". This study is grounded in deep mid-IR (IRAC) data, and we are most sensitive to the more embedded Stage I sources. This is also likely a physical effect in part resulting from faster evolution of later stages of protostellar evolution (e.g., W08 and Carlson et al. 2011). Evolutionary stages as used here are reasonably well-correlated with the Classes I, II, and III of Lada (1987) and Types I, II, and III from Chen et al. (2009).

\section{Application to nine star-forming regions}

We apply the YSO selection criteria described in Sect. 3 to LMC star formation regions N11, N44, N51, N105, N113, N120, $\mathrm{N} 144$, N160, and N206. These nine regions are selected to cover a range of sizes, morphologies, and levels of star formation activity. Figure 4 shows their distribution within the LMC and with the exact areas we study. Of more than 66000 SAGE sources within these regions, $\sim 5300$ meet our color-selection criteria for sets 1-9, and we ultimately consider 1045 to be well-fit YSO candidates.
We report 918 entirely new candidate YSOs here. We consider 1045 sources to be well-fit YSOs, 127 of which are also selected by W08, GC09, Chen et al. (2009), Romita et al. (2010), Chen et al. (2010), Fleener et al. (2010), or some combination. For completeness, our images and photometric catalog include another 358 YSO candidates from those lists, most of which do meet our color-selection criteria but are not well fit by YSO models. We do assign approximate evolutionary stages from the fitter for use in images; we make no cuts on the basis of fit quality and make no claims about their physical characteristics. Combining our list of well-fit sources with the candidate YSOs from the other 5 lists, we consider 1403 sources. A detailed comparison of the source lists is presented in Sect. 5.1 and Table 7. Visually, we find that our new candidates align well with previously identified candidates, spectroscopically "confirmed" YSOs, and the physical structure of the ISM.

We determine approximate physical parameters for each "well-fit" source, averaging parameters of all YSO models with SEDs in the range $\chi^{2} / \mathrm{pt} \leq \chi_{\min }^{2} / \mathrm{pt}+1$. Resulting parameters are listed in Table 5. For each source, we provide the range of $\chi^{2} / \mathrm{pt}$ values used, the number of models fit within that range, the derived evolutionary stage, and the physical parameters of the YSO models: stellar mass, stellar luminosity, envelope accretion rate, disk mass, and envelope mass.

Table 6 shows the breakdown of the evolutionary stages of our well-fit sources in each region. Approximately $56 \%$ of all of our YSOs are classified as Stage I, 12\% as Stage I/II, $23 \%$ as Stage II, and only $\sim 9 \%$ as Stage II/III, Stage III, or Unclassified. This is at least partially an effect of our sensitivity. Stage I sources are brightest in the mid-IR. Interestingly, $81 \%$ of N160 sources are Stage I or Stage I/II, compared to just over $70 \%$ in $\mathrm{N} 105$ and $62-66 \%$ in the other 7 regions.

\subsection{N11}

Located in the north-west corner of the LMC, N11 is one of the most well-studied star-forming regions in the LMC. The nebula is second in size and $\mathrm{H} \alpha$ luminosity only to the famous ministarburst 30 Doradus on the other side of the galaxy (Walborn \& Parker 1992). It displays a central cavity, relatively devoid of IR emission, surrounded by a complex structure of HII regions of radius $\sim 100 \mathrm{pc}$, described as a "ring of HII regions" by Rosado et al. (1996). The young stellar association LH 9 (Lucke \& Hodge 1970) has cleared the central cavity with winds of its massive stars, creating diffuse soft X-ray emission (Nazé et al. 2004), and triggering star formation around the bordering regions. In addition to star formation immediately around the central bubble (notably, abundant Herbig Ae/Be stars encircling the LH 9 cavity, following the $\mathrm{H} \alpha$ ridge; Hatano et al. 2006 , from IRSF observations), star formation is propagating outward through the surrounding nebula. N11B is one of the secondary nebulae (the $24 \mu \mathrm{m}$-bright region on the north-east edge of the central cavity in Fig. 5) and contains the embedded stellar association LH 10 and the star-formation signpost of a 6.7 GHz methanol maser MC18 (Ellingsen et al. 1994; Barbá et al. 2003; Green et al. 2008; Ellingsen et al. 2010), which is also a spectroscopically confirmed YSO (Seale et al. 2009). Three X-ray point sources and excess X-ray emission, probably indicating the presence of young T-Tauri stars (Nazé et al. 2004). Vallenari et al. (2010) find further evidence of sequential star formation from the distribution of their YSO candidates. Using the same "Types" as Chen et al. (2009), they find that 


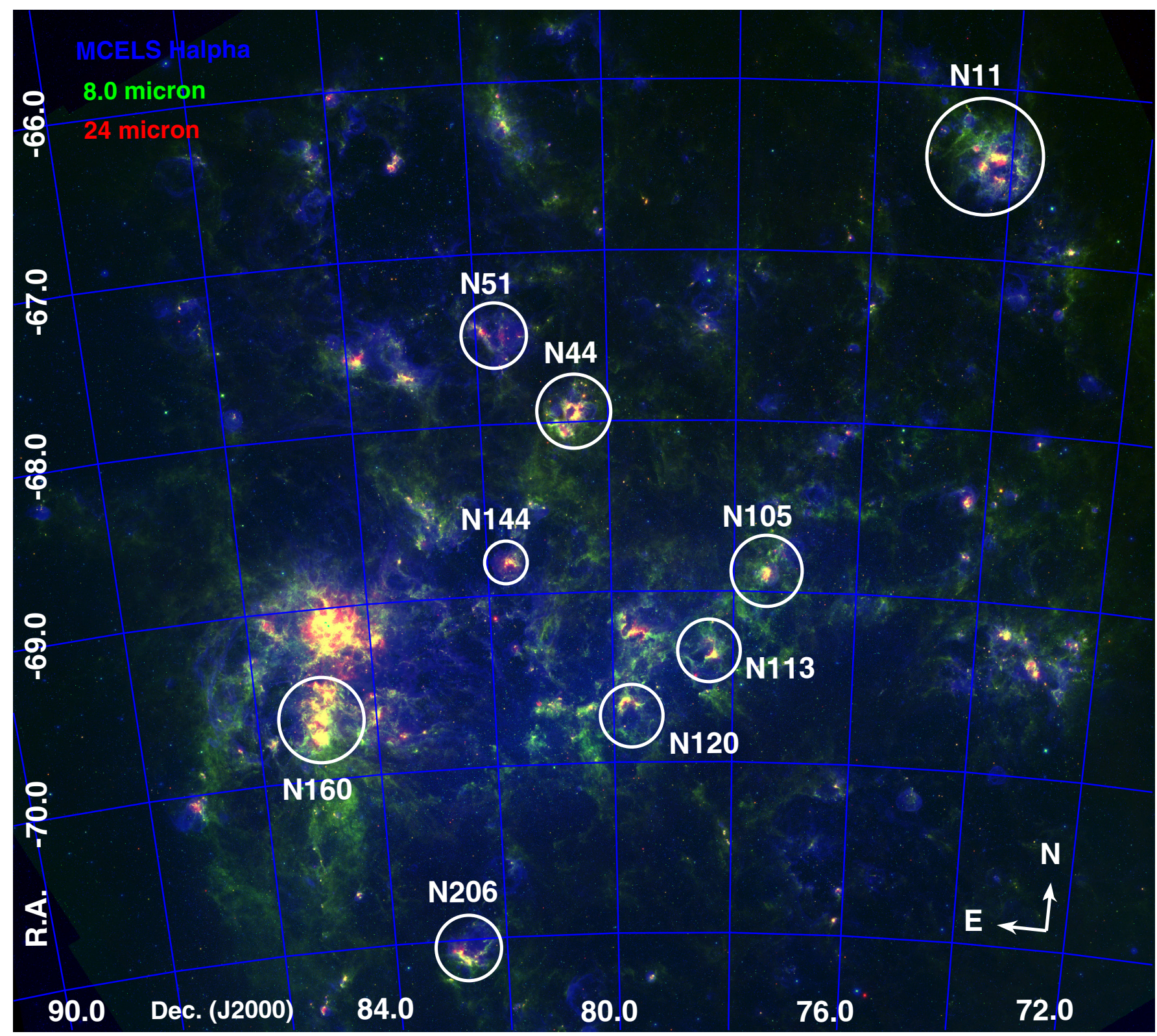

Fig. 4. Examined star-forming regions on MCELS H $\alpha$ (blue) + SAGE $8 \mu$ m (green) $+24 \mu \mathrm{m}$ (red). H $\alpha$ image from the Magellanic Cloud Emission Line Survey (MCELS; Smith \& MCELS Team 1998; Smith et al. 2005).

Type II are preferentially close to LH 10, and Type I are even farther from LH 9.

Although age estimates vary, several authors report a marked difference in the ages of LH 9 and LH 10, suggesting that the LH 9 has triggered the current star formation in LH 10 and in other surrounding nebular bubbles. Walborn et al. (1999) estimate the triggering difference to be $\delta_{\mathrm{t}} \sim 2 \mathrm{Myr}$ and estimate ages of 3.5 and 1 Myr for LH 9 and LH 10, respectively, based on HST optical imaging. Massey et al. (2000) examine the most massive stars both photometrically and spectroscopically and report that all stars in LH 9 with masses greater than $20 M_{\odot}$ are approximately coeval with an age of $\sim 4 \mathrm{Myr}$ and a turn-off mass of $\sim 45 M_{\odot}$. Age estimates from Mokiem et al. (2007) are significantly older at $7 \pm 1 \mathrm{Myr}$ and $3 \pm 1 \mathrm{Myr}$ but still indicate sequential formation. Vallenari et al. (2010) examine both HST/ACS and Spitzer data to identify a significant optical PMS population and 30 color-selected YSO candidates with HST PMS stars and protoclusters physically mixed. They find that the PMS stars intermixed with MS stars in the central cavity, with LH 9 populations in the 3-10 Myr age range and the main PMS population $\sim 5$ Myr old. They estimate the LH 10 association age as $3 \pm 1$ Myr. Heydari-Malayeri et al. (2000) and Heydari-Malayeri et al. (2001) find similar results based on spectroscopy of blue stars in N11B.

We consider a circular area of radius 20.5 (nearly 300 pc or an area of $0.28 \mathrm{kpc}^{2}$ at the distance of the LMC), centered at $04^{\mathrm{h}} 56^{\mathrm{m}} 48^{\mathrm{s}},-66^{\circ} 24^{\prime} 00^{\prime \prime}$. Seventy-four candidate YSOs were identified within this area by W08 and/or GC09. Seale et al. (2009) confirmed the YSO nature of 32 sources using Spitzer/IRS spectroscopy, and Woods et al. (2011) confirmed one more. Of the Seale et al. (2009) sources, thirty-one were chosen from the GC09 list, and the last corresponds to the methanol maser. We identify 242 well-fit YSO candidates in the region, 218 of which are on no previous list. We find that star formation is continuing throughout the region with particular concentration along filaments and near the edges of photodissociation regions. 


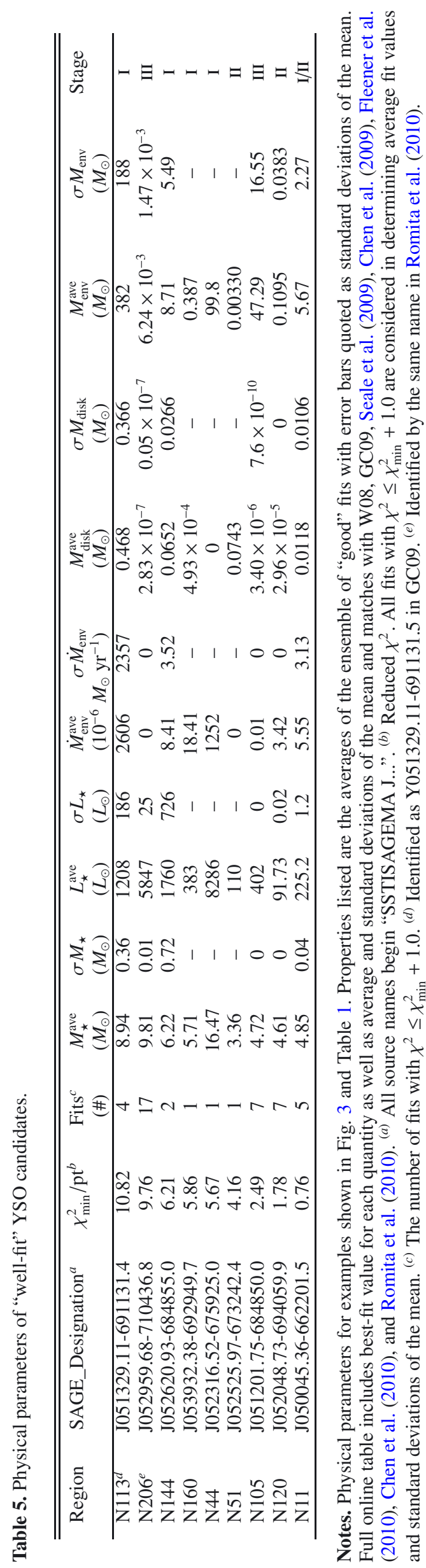

\subsection{N44}

Along with 30 Doradus and N11, N44 is one of the most active star-forming regions in the LMC, injecting vast amounts of energy into the ISM. Located in north of the LMC bar, N44 is characterized by interacting shells of star formation. The most prominent feature is Shell 1 (Chen et al. 2009), the central bubble in our Fig. 6, which encircles the LH 47 cluster of massive, ionizing stars (Stasińska et al. 1986; Meaburn \& Laspias 1991). Will et al. (1997) estimate the age of LH 47 at 5-6 Myr, based on a combination of photometry and spectroscopy. Oey \& Massey (1995) note two populations, one $\gtrsim 10$ Myr inside Shell 1 and one outside, resulting from a burst of star formation in the last $\sim 5$ Myr. Shell 1 is expanding spherically (Meaburn \& Laspias 1991; Chu et al. 1993) with a dynamical age greater than $1 \mathrm{Myr}$, evidently triggering star formation along the edges, as can be seen in Fig. 6. HI maps suggest similar scenarios for the other HI shells in the area; compression from interacting shells expanding into one another is triggering star formation (Kim et al. 1998). Massey et al. (2000) classify one Nitrogen rich W-R star in LH 47 and estimate an age of $\sim 2.95$ Myr for all LH 47 stars with $M>20 M_{\odot}$ and an age of $\sim 2.14$ Myr for the 3 most massive stars. Exterior to (i.e., around the rim of) the superbubble, they estimate a turn-off mass of $\sim 55 M_{\odot}$.

Chen et al. (2009) present a detailed study of the massive YSO population in N44 and note evidence of triggered star formation. They identify 99 candidate YSOs, using the same colorselection criteria as GC09, then narrow their list to 60 probable YSOs via visual inspection of SEDs and imaging. They use deep IRAC and MIPS imaging and add NIR and optical imaging and photometry. MCELS $\mathrm{H} \alpha$ and sundry optical archival HST images are used for image examination. They fit SEDs of 36 YSO candidates judged to be unambiguously single or dominant sources. Using the grid of Robitaille et al. (2006) as a guide, they then classify each of these sources into Types I-III by visual examination rather than relying upon the physical characteristics suggested by the YSO model grid. They also report the range of Robitaille et al. (2006) stages found and find that stage and type are equivalent for most sources.

The area we study, a circle of radius 13.5 centered at $\mathrm{RA}=05^{\mathrm{h}} 22^{\mathrm{m}} 07^{\mathrm{s}}, \mathrm{Dec}=-67^{\circ} 56^{\prime} 24^{\prime \prime}$ and covering $0.11 \mathrm{kpc}^{2}$ in the LMC, includes 32 spectroscopically confirmed YSOs from Seale et al. (2009), many occurring in clumps with several photometric YSO candidates. Star formation is highly concentrated in shell-like lanes or ridges. We find a total of 139 well-fit YSO candidates, 24 of which appear in at least one previous list. Figure 6 shows the distribution of candidates, including 74 previously identified by W08, GC09, and/or Chen et al. (2009) and 115 new candidates.

\subsection{N51}

In the northern disk, the N51 star-forming region is characterized by lanes of active star formation along the boundaries between expanding superbubbles. Superbubble N51D surrounds OB associations LH51 and LH54 (Lucke \& Hodge 1970; Oey \& Smedley 1998). Meaburn \& Terrett (1980) use [O II] emission line profiles to show that N51D is expanding at $\sim 35 \mathrm{~km} \mathrm{~s}^{-1}$ toward the observer and at $\sim 25 \mathrm{~km} \mathrm{~s}^{-1}$ away from the observer, possibly into a denser medium. Expansion is likely driven by a supernova remnant or possibly energetic winds of enclosed massive stars. Chu et al. (2005) identify 3 YSO candidates in N51D. HST emission line images show that 2 of the YSO candidates are associated with Bok globules, and the 


\section{R. Carlson et al.: LMC YSO selection}

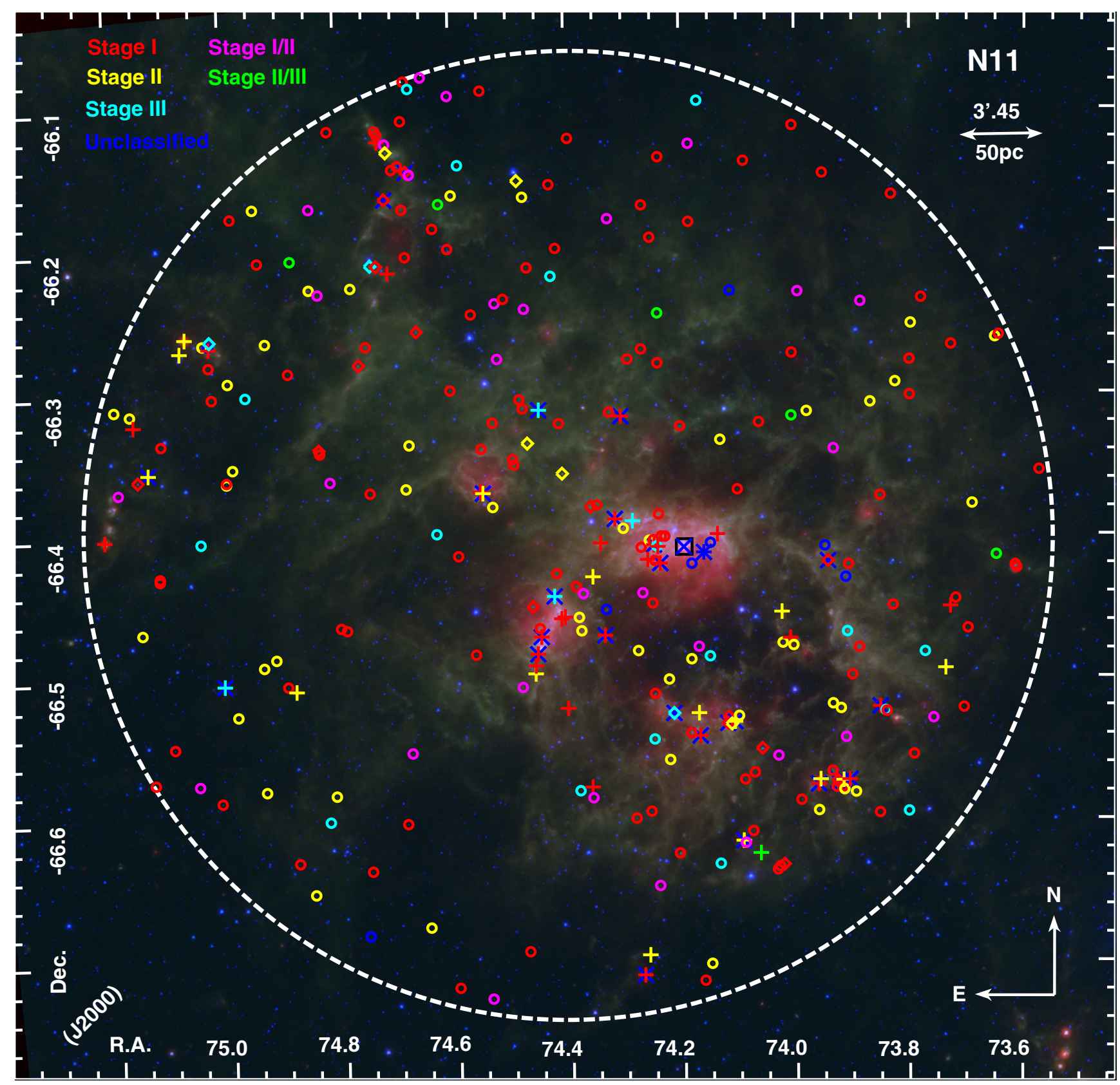

Fig. 5. Three-color image of N11 with YSO candidates marked. Full region in IRAC $3.6 \mu \mathrm{m}$ (blue) $+8.0 \mu \mathrm{m}$ (green) + MIPS $24 \mu \mathrm{m}$ (red). Candidate YSOs are marked in red for Stage I, magenta for Stage I/II, yellow for Stage II, green for Stage II/III, cyan for Stage III, and blue for unclassified. Circles are newly identified candidates. Crosses are from W08 and/or GC09. Diamonds indicate sources on our list that are also in another list, and blue Xs are spectroscopically confirmed YSOs from Seale et al. (2009) or Woods et al. (2011).

Table 6. LMC YSO candidate evolutionary stages.

\begin{tabular}{lccccccc}
\hline \hline Region & Stage I & Stage I/II & Stage II & Stage II/III & Stage III & Unclassified & Total \\
\hline N11 & 126 & 32 & 53 & 4 & 20 & 7 & 242 \\
N44 & 66 & 21 & 36 & 2 & 11 & 3 & 139 \\
N51 & 54 & 14 & 23 & 2 & 10 & 1 & $103^{a}$ \\
N105 & 51 & 13 & 20 & 1 & 4 & 2 & 91 \\
N113 & 53 & 12 & 30 & - & 6 & - & 101 \\
N120 & 22 & 10 & 15 & 1 & 1 & 2 & 51 \\
N144 & 18 & 2 & 6 & - & 2 & 3 & 31 \\
N160 & 155 & 16 & 32 & 4 & 4 & 3 & 214 \\
N206 & 39 & 9 & 20 & 1 & 2 & 2 & 73 \\
\hline Totals & 584 & 129 & 235 & 14 & 60 & 23 & 1045 \\
\hline
\end{tabular}

Notes. ${ }^{(a)}$ Two more N51 "well-fit" YSO candidates are readily identifiable as parts of a background galaxy, one is fit as Stage I and one as Stage III. 


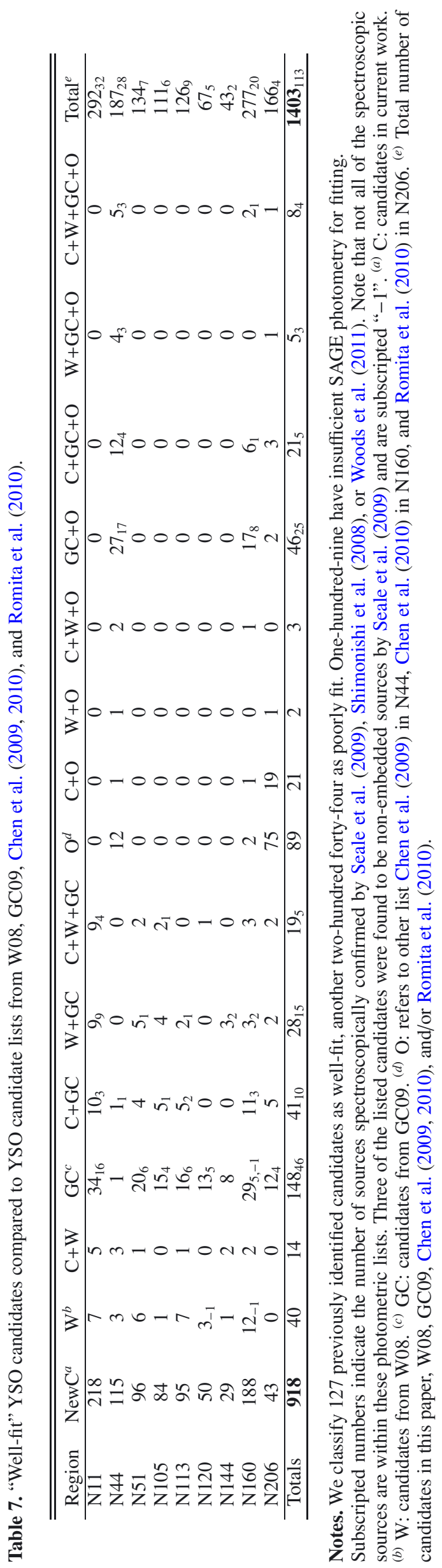

morphology and the $\mathrm{H} \alpha /[\mathrm{SII}]$ ratio of the third suggests that it may be a Herbig-Haro object. From the models of Whitney et al. (2003a), Chu et al. (2005) estimate all three sources to have masses in the B star range. Breysacher et al. (1999) report HD 36502 in LH54 as a binary or trinary system WC4(+O)+O8I (spectroscopy from Moffat et al. 1990), again supporting the young age of the association.

The area considered here is a circle of radius 11.5 $\left(167.5 \mathrm{pc}\right.$; area $\left.0.087 \mathrm{kpc}^{2}\right)$ centered at RA $=05^{\mathrm{h}} 26^{\mathrm{m}} 53^{\mathrm{s}}$, Dec $=-67^{\circ} 28^{\prime} 48^{\prime \prime}$. There are 38 YSO candidates from W08 and/or GC09, including seven spectroscopically confirmed by Seale et al. (2009). We consider seven of these to be well fit and identify 96 new candidates tracing the same ridges of star formation.

\subsection{N105}

N105 is an average-sized HII region, situated in a quiescent part of the LMC at the western side of the bar. The molecular cloud is still being evaporated, creating an inhomogeneous region of dust globules and pillars surrounded by hot gas. At the center of N105 sits the OB association LH 31 (corresponding to the strongest X-ray emission Dunne et al. 2001), which includes 2 W-R stars. Massey et al. (2000) find that the young (optical) stellar population is not co-eval; the 3 most massive are the youngest $(\sim 1.5 \mathrm{Myr})$, but the population of stars with $M>20 M_{\odot}$ are $\sim 3.5 \mathrm{Myr}$ in age. They estimate the turn-off mass of these massive stars to be $\sim 65 M_{\odot}$, but note that there are some evolved stars with masses $\sim 15-40 M_{\odot}$, indicating either ongoing or somewhat episodic star formation. The densest central region, N105A, shows limited evidence for massive star feedback; no shell structure is seen in OIII images (Ambrocio-Cruz et al. 1998), in spite of its proximity to LH 31. Star formation is ongoing deep in its core with a massive spectroscopic YSO identified by Epchtein et al. (1984) and Oliveira et al. (2006) and several maser sources (water, $\mathrm{OH}$, and methanol, Sinclair et al. 1992; Lazendic et al. 2002; Oliveira et al. 2006; Green et al. 2008; Ellingsen et al. 2010). A cluster of four compact radio continuum sources has also been detected (Indebetouw et al. 2004) in this region, signposts of young embedded ultra-compact HII regions.

We cover both the dense N105A region and its diffuse surroundings, looking at a circle of radius $12.5\left(181 \mathrm{pc} ; 0.10 \mathrm{kpc}^{2}\right.$ in area) centered at $\mathrm{RA}=05^{\mathrm{h}} 09^{\mathrm{m}} 53^{\mathrm{s}}$, Dec $=-68^{\circ} 52^{\prime} 48^{\prime \prime}$. We find 91 well-fit YSO candidates, eighty-four of which are new and seven of which are also in the GC09 list or both the W08 and GC09 lists. In Fig. 8, we also mark another 20 sources from the W08 and/or GC09 lists, six spectroscopic YSOs from Seale et al. (2009), and the masers mentioned above.

\section{5. $N 113$}

The H II region N113 falls in the middle of the LMC bar. It shows a complex structure of $\mathrm{H} \alpha$ bubbles with a rich molecular gas and dust morphology. Several young clusters (<10 Myr; NGC 1874, NGC 1876, and NGC 1877) are associated with N113 (Bica et al. 1996) and massive stars in these clusters are responsible for the several ionized gas bubbles that compress the remnant molecular material (Oliveira et al. 2006). In this compressed dense lane of neutral gas and dust star formation is occurring, pinpointed by maser sources in its earliest stages and by the continuum emission as the massive stars evolve (Brooks \& Whiteoak 1997; Wong et al. 2006). This region also hosts the most intense 


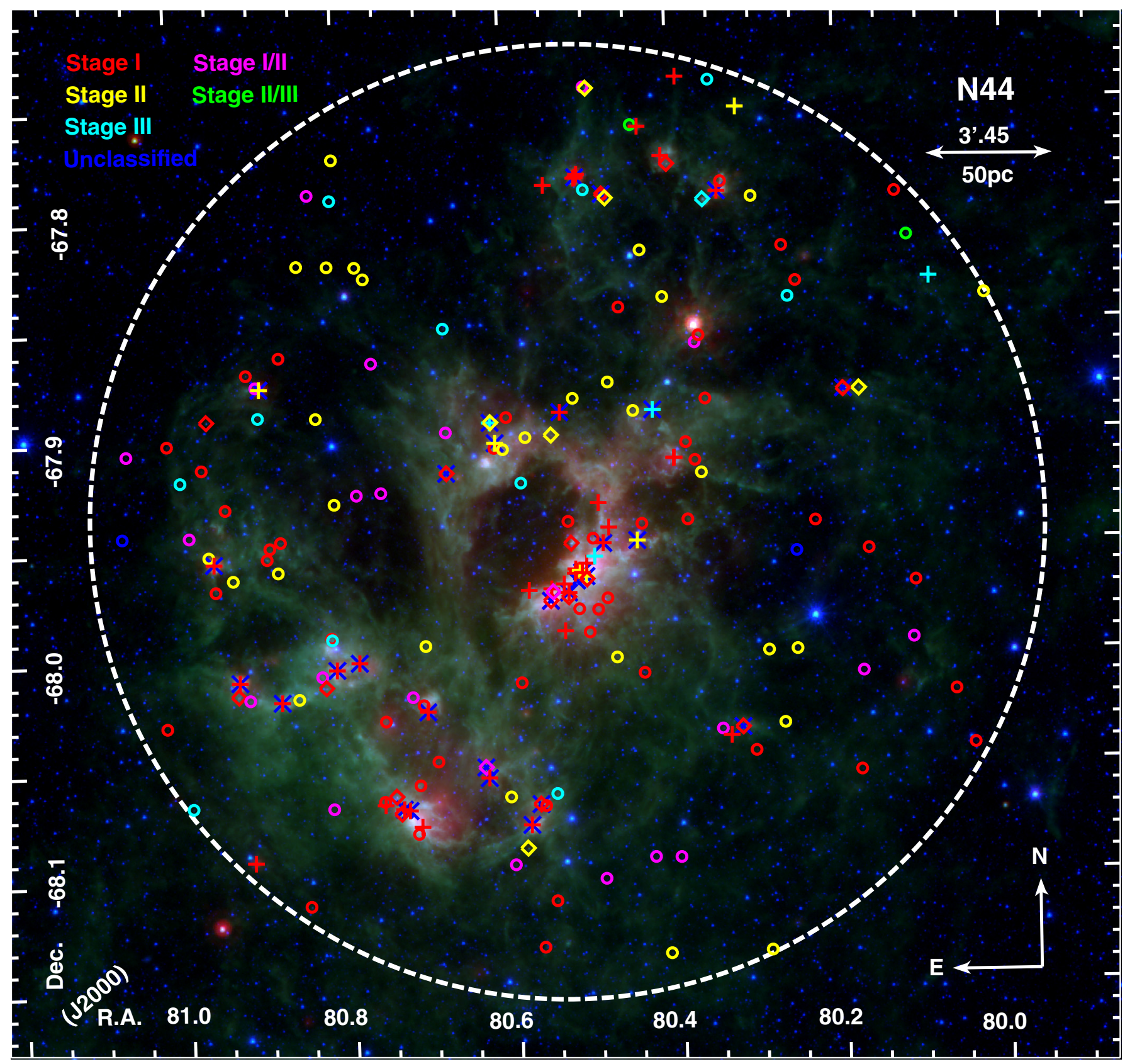

Fig. 6. Three-color image of N44 with YSO candidates marked. Full region in IRAC $3.6 \mu \mathrm{m}$ (blue) $+8.0 \mu \mathrm{m}$ (green) + MIPS $24 \mu \mathrm{m}$ (red). Circles are newly identified candidates. Crosses are from W08, GC09, and/or Chen et al. (2009). Diamonds indicate sources on our list that are also in another list, and blue Xs are spectroscopically confirmed YSOs from Seale et al. (2009).

$\mathrm{H}_{2} \mathrm{O}$ maser $^{3}$ of the Magellanic Clouds (Whiteoak \& Gardner 1986; Lazendic et al. 2002; Oliveira et al. 2006) as well as an OH maser ${ }^{4}$ (Brooks \& Whiteoak 1997), both signposts of the early stages of star formation. N113 therefore presents a clear example of star formation triggered by the winds from massive stars (Oliveira et al. 2006). We highlighted this region in our Herschel YSO demonstration paper (Sewiło et al. 2010), showing that there are at least 9 far-IR YSO candidates, even more embedded than Stage I sources presented here.

We center our study at RA $=05^{\mathrm{h}} 13^{\mathrm{m}} 36^{\mathrm{s}}$, Dec $=-69^{\circ} 21^{\prime} 00^{\prime \prime}$, covering an area of radius $11^{\prime}\left(160 \mathrm{pc}\right.$ in radius or $0.080 \mathrm{kpc}^{2}$ in area). We find 95 new YSO candidates and find six

\footnotetext{
$\mathrm{H}_{2} \mathrm{O}$ maser: $22 \mathrm{GHz}$.

4 OH maser: 1665-MHz.
}

previously-identified candidates to be well-fit. In Fig. 9, we also mark 25 candidates from W08 and GC09 that do not meet our selection criteria. There are 9 spectroscopically "confirmed" YSOs (Seale et al. 2009) in this region. Two are in our list and in the GC09 list, while six are in the GC09 list and one in both W08 and GC09.

\section{6. $N 120$}

The third of our medium-sized star-forming regions in the LMC's stellar bar is N120. It is an HII region of deceptively simple ring morphology. The ionization front is associated with interior stars, such as those in OB association LH 42 (Lucke \& Hodge 1970). It also includes a supernova remnant (identified 


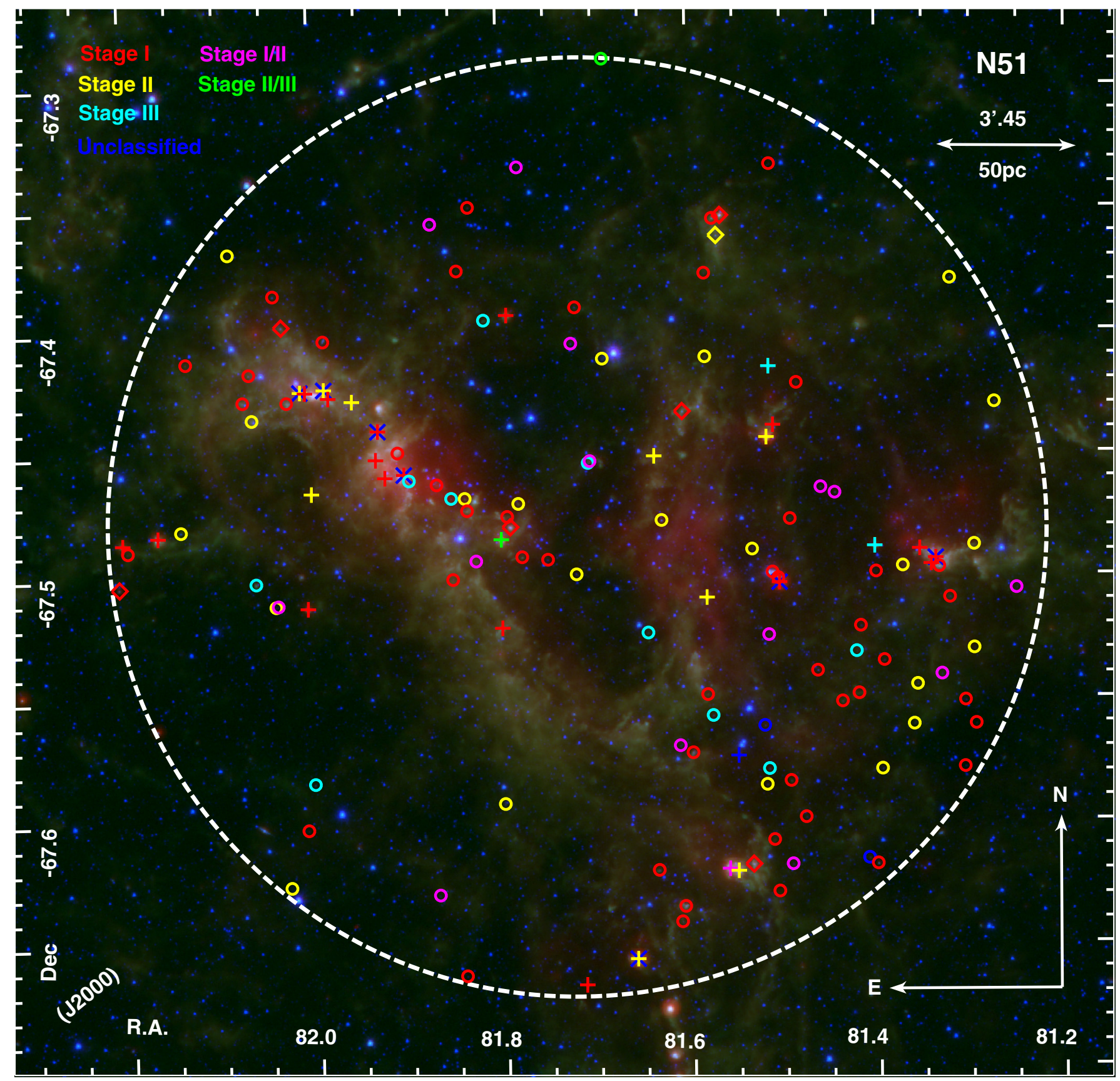

Fig. 7. Full N51 region in IRAC $3.6 \mu \mathrm{m}$ (blue) $+8.0 \mu \mathrm{m}$ (green) + MIPS $24 \mu \mathrm{m}$ (red). Candidate YSOs are marked as in Fig. 5. Circles are newly identified candidates. Crosses are from W08 and/or GC09. Diamonds indicate sources on our list that are also in another list, and blue Xs are spectroscopically confirmed YSOs from Seale et al. (2009).

via strong [SII] and [OI] lines; Danziger \& Dennefeld 1974). Two wind-blown bubbles join together to form the incomplete ring. The hottest stars are not centrally located, and their winds and ionizing radiation serve to drive violent motion at the ring's boundaries (Laval et al. 1992).

We consider an area of radius $11^{\prime}(\sim 160 \mathrm{pc})$ centered at $\mathrm{RA}=05^{\mathrm{h}} 18^{\mathrm{m}} 48^{\mathrm{s}}$, Dec $=-69^{\circ} 43^{\prime} 48^{\prime \prime}(\mathrm{J} 2000)$. At the distance of the LMC, this is an area of $0.080 \mathrm{kpc}^{2}$. We identify 51 YSO candidates, 50 of which are newly identified, following the dust ridges as seen in Spitzer images (Fig. 10). We also mark 13 further candidates from GC09 and 3 from W08. Five spectroscopic YSOs from Seale et al. (2009) are in this region, four of which are in the GC09 list and one in both the GC09 list and ours. One W08 source was classified as a non-embedded source, possibly an O-rich AGB, by Seale et al. (2009).

\subsection{N144}

The little-studied N144 HII region is located North of the LMC bar and West of the massive 30 Dor complex, on the edge of the super giant shell LMC 3 and is notable for its strong $24 \mu \mathrm{m}$ emission. Massey et al. (2000) find three W-R stars in the central region (LH 58) and estimate an optical turn-off mass of $\sim 50 M_{\odot}$. For the stars of mass greater than $20 M_{\odot}$, they give $\$ 3 \mathrm{Myr}$, and find the most massive stars to be coeval, though 


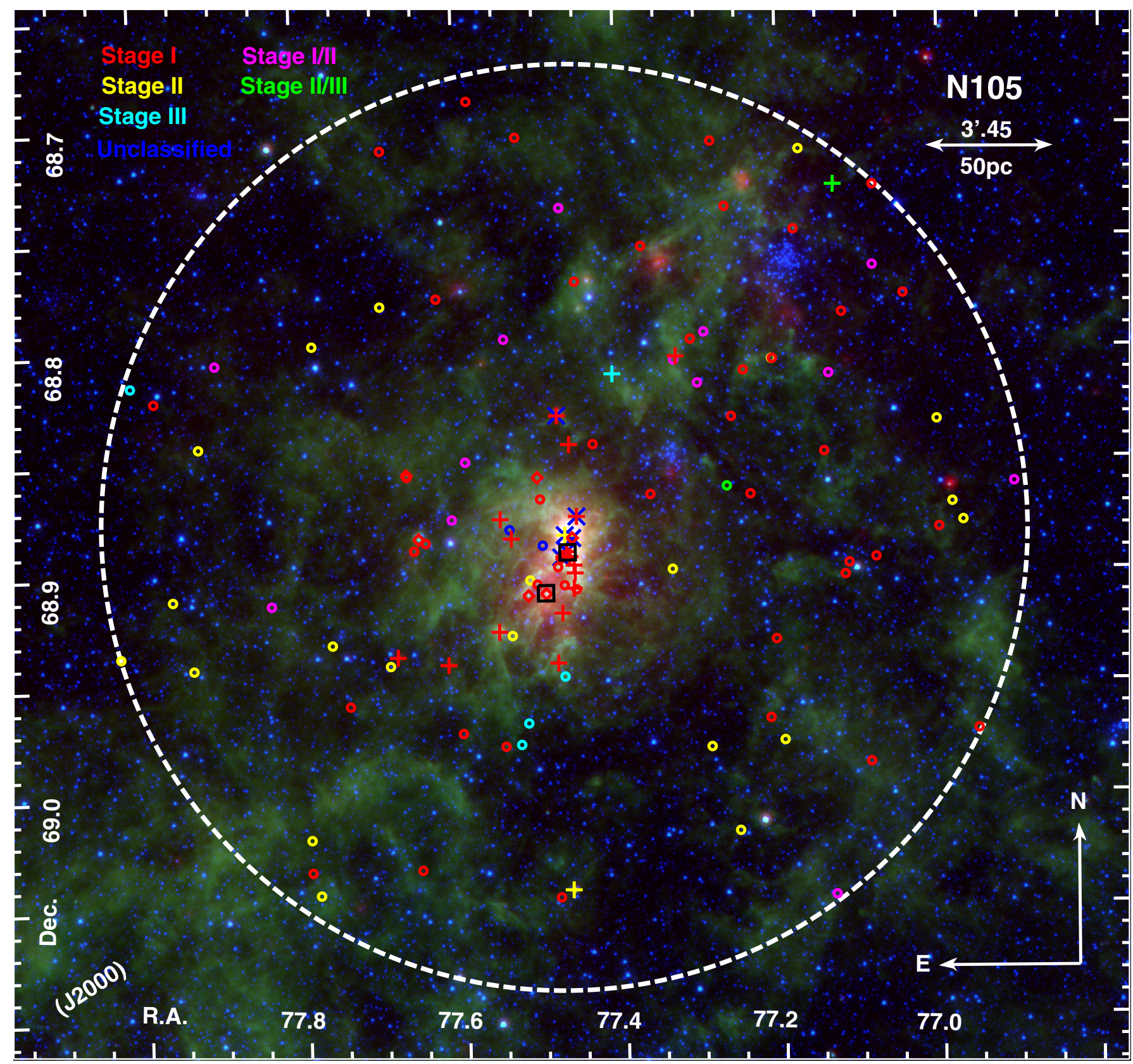

Fig. 8. Three-color image of N105 with YSO candidates marked. Full region in IRAC $3.6 \mu \mathrm{m}$ (blue) $+8.0 \mu \mathrm{m}$ (green) + MIPS $24 \mu \mathrm{m}$ (red) Candidate YSOs are marked as in Fig. 5: circles are new candidates, crosses are from W08 and/or GC09, diamonds are in our list and at least one other. Blue Xs are spectroscopically confirmed YSOs from Seale et al. (2009). Large squares show masers from Sinclair et al. (1992); Lazendic et al. (2002); Oliveira et al. (2006); Ellingsen et al. (2010), as described above.

Lortet \& Testor (1988) note that the UV/optical stellar population seems to be somewhat older in the south-east than the north.

We inspect an area of radius 7.5 centered at $\mathrm{RA}=$ $05^{\mathrm{h}} 26^{\mathrm{m}} 47^{\mathrm{s}}$, Dec $=-68^{\circ} 48^{\prime} 48^{\prime \prime}(\mathrm{J} 2000)$. At the distance of the LMC, this is an area of $0.037 \mathrm{kpc}^{2}$. Fourteen YSO candidates were identified in this region by W08 and/or GC09. One was spectroscopically confirmed by Seale et al. (2009) and another by Woods et al. (2011). We identify 31 YSO candidates as well-fit, 29 of them newly identified (Fig. 11).

\subsection{N160}

The region for which we use the shorthand N160 here actually encompasses a large section of the molecular ridge south of 30 Dor, including N155, N159 (subject of recent YSO discovery paper Chen et al. 2010), N172, N173, and part of N158. HII regions in this molecular ridge have a significantly higher CO content than that of 30 Dor itself (Johansson et al. 1998), indicative of early stages of star formation. Fariña et al. (2009) spectroscopically classify over $150 \mathrm{O}$ and early B-type stars in the region. Part of the extended 30 Doradus complex, various sub-regions have been extensively studied (cf. Israel et al. 1996; Nakajima et al. 2005; Nakajima 2005, and many others). Chen et al. (2010) present a detailed examination of the subregion N159, beginning with the YSO selection techniques of GC09 and adding high resolution NIR imaging. They find that many Spitzer YSO candidates are actually compact clusters or multiple sources, and some of their color-selected candidates 


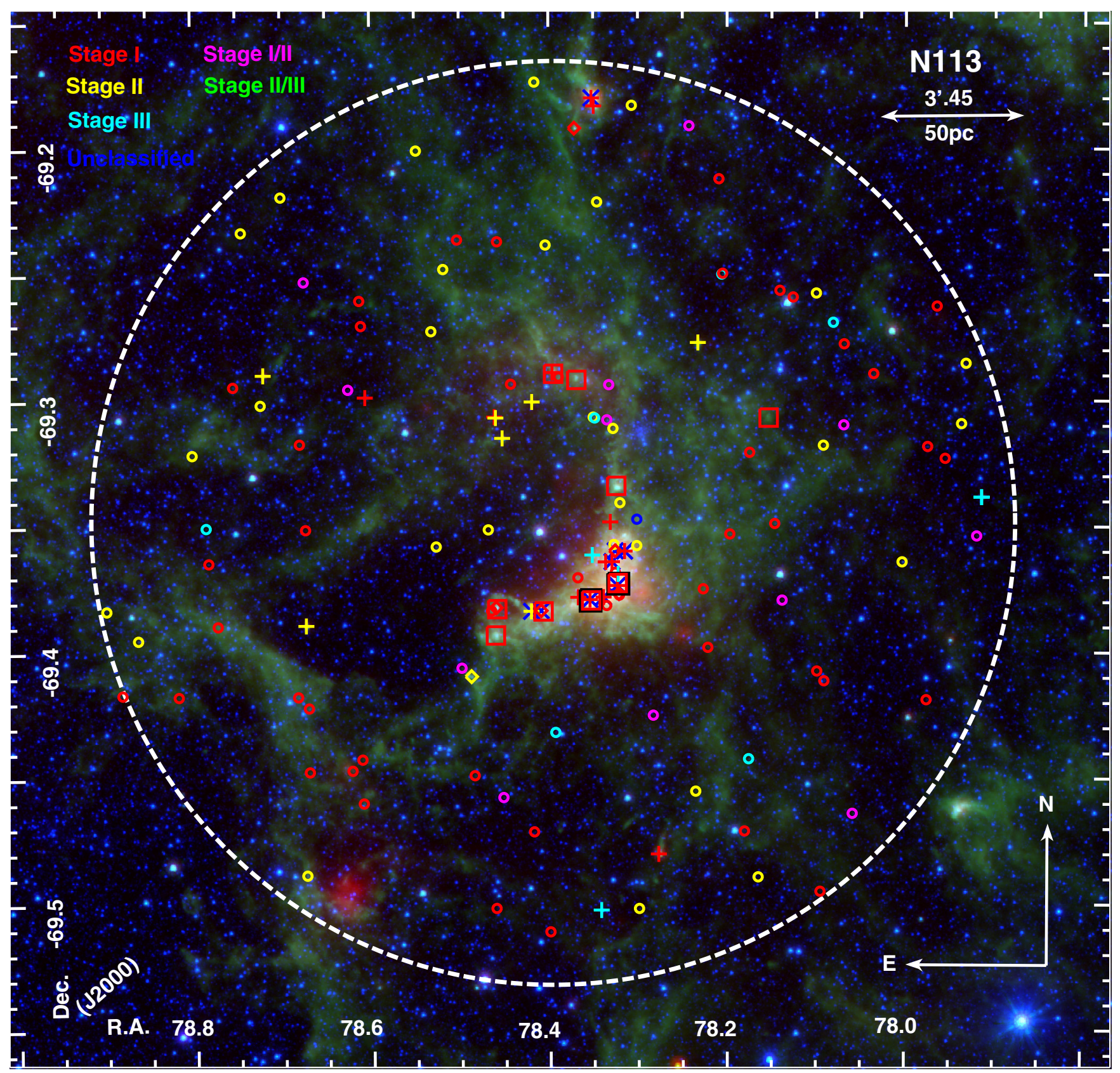

Fig. 9. Three-color image of N113 with YSO candidates marked. Full region in IRAC $3.6 \mu \mathrm{m}$ (blue) $+8.0 \mu \mathrm{m}$ (green) + MIPS $24 \mu \mathrm{m}$ (red). Candidate YSOs are marked as in Fig. 5. Red squares indicate the locations of Herschel YSO candidates from Sewiło et al. (2010). Black squares show masers from Brooks \& Whiteoak (1997); Whiteoak \& Gardner (1986); Lazendic et al. (2002); Oliveira et al. (2006), as described above.

are probably MS stars in front of bright dust. Similarly, Fleener et al. (2010) study high mass star formation in NGC 2074 and find 5 of the 10 GC09 YSO candidates show signs of multiplicity. Three 22-GHz water masers have also been observed. Two of these are in the NGC 2080 region, one in combination with $6.035-\mathrm{GHz} \mathrm{OH}$ maser and one in combination with 6.7-GHz methanol Ellingsen et al. (2010).

This is the second largest region we inspect, covering an area of $0.15 \mathrm{kpc}^{2}$ (radius $15^{\prime} ; 217.5 \mathrm{pc}$ ) centered at RA = $05^{\mathrm{h}} 39^{\mathrm{m}} 44^{\mathrm{s}}$, Dec $=-69^{\circ} 38^{\prime} 00^{\prime \prime}(\mathrm{J} 2000)$. We find 214 well-fit YSO candidates and mark another 63 from W08, GC09, and Chen et al. (2010) in Fig. 12. Twenty-six of our well-fit sources are also on at least one other list. Also in the region covered here are 28 spectroscopically "confirmed" YSOs, twenty-seven from Seale et al. (2009) and one from Shimonishi et al. (2008).
One candidate from W08 and one from GC09 were found to be non-embedded sources by Seale et al. (2009).

\subsection{N206}

Southwest of 30 Dor and N160, N206 is relatively isolated in the disk of the galaxy. It is characterized by a dense molecular ridge surrounding an evacuated shell. Kim et al. (1998) list two expanding HI shells (GS 69 and GS 70), the edges of which correspond to the dusty ridges. Active star formation is continuing throughout the region, concentrated along the ridges as shown by Romita et al. (2010) in a detailed study of the region using the same imaging and photometry used here. They identified more than 100 candidate YSOs and estimated a SFR of $2.7 \times 10^{-2} M_{\odot} \mathrm{yr}^{-1} \mathrm{kpc}^{-2}$. 


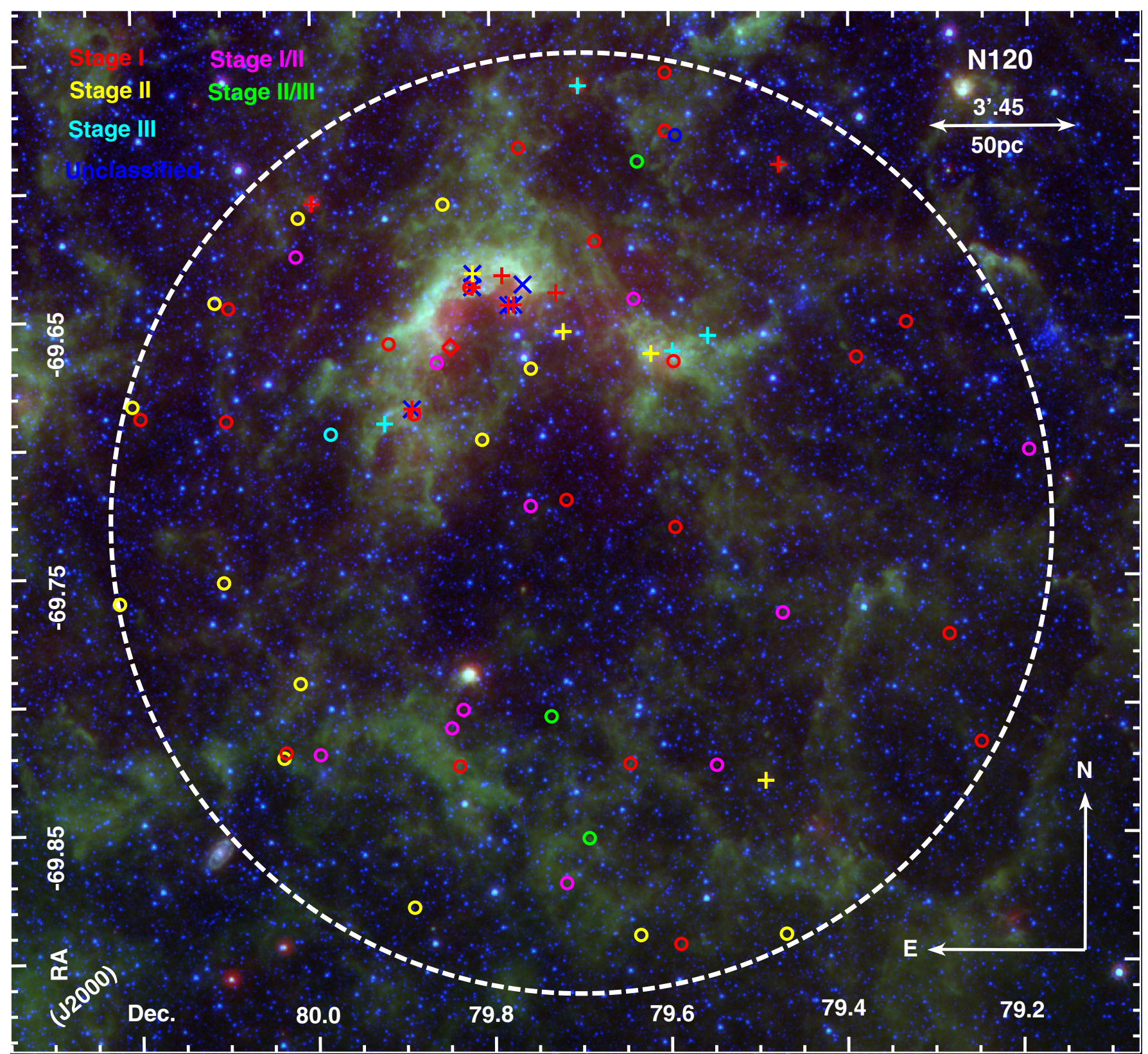

Fig. 10. Three-color image of N120 with YSO candidates marked on IRAC $3.6 \mu \mathrm{m}$ (blue) $+8.0 \mu \mathrm{m}$ (green) + MIPS $24 \mu \mathrm{m}$ (red). Candidate YSOs are marked as in Fig. 5: circles - new candidates, crosses - W08/GC09 candidates, diamonds - previously identified candidates on our list. Blue Xs are spectroscopic YSOs from Seale et al. (2009).

We consider the same area as Romita et al. (2010), a circle of radius 11:5 (167.5 pc) centered at $\mathrm{RA}=05^{\mathrm{h}} 30^{\mathrm{m}} 48^{\mathrm{s}}$, Dec $=$ $-71^{\circ} 03^{\prime} 00^{\prime \prime}$. We find 73 well-fit YSO candidates, 43 of which are newly identified. In Fig. 13, we also mark 93 candidates from W08, GC09, and Romita et al. (2010) that we do not consider well-fit. Seventy-five candidates are only in the Romita et al. (2010) list, which was constructed via a similar technique to that used here; see Sect. 5.1 for details. Four Seale et al. (2009) spectroscopic sources are in the GC09 list.

\section{Results}

\subsection{Comparison to previous lists}

Our YSO candidate selection reproduces previous lists well. While only 127 of the 485 YSO candidates identified by W08,
GC09, Chen et al. (2009), Romita et al. (2010), and Chen et al. (2010) meet our final fitting criteria, only two sources do not fall within our color-cuts, both from Romita et al. (2010). Alternative names and classifications for all sources from W08, GC09, Seale et al. (2009), Chen et al. (2009), Romita et al. (2010), Fleener et al. (2010), and Chen et al. (2010) are presented in Table 3.

One-hundred-nine candidates from GC09 and/or Chen et al. $(2009,2010)$ are not in our SAGE catalog at all or are but have insufficient photometry for our color-selection process. These are preferentially bright or extended sources that do not fulfill our strict PSF photometry. Based on their native photometry, all would fit our color criteria: forty-nine in Set 1, four in Set 2, forty-eight in Set 3, two in Set 4, three in Sets 5, one in Set 6, and two in 8. We do not perform careful SED fitting on these sources but do a cursory treatment to assign evolutionary stages for plotting on images. 


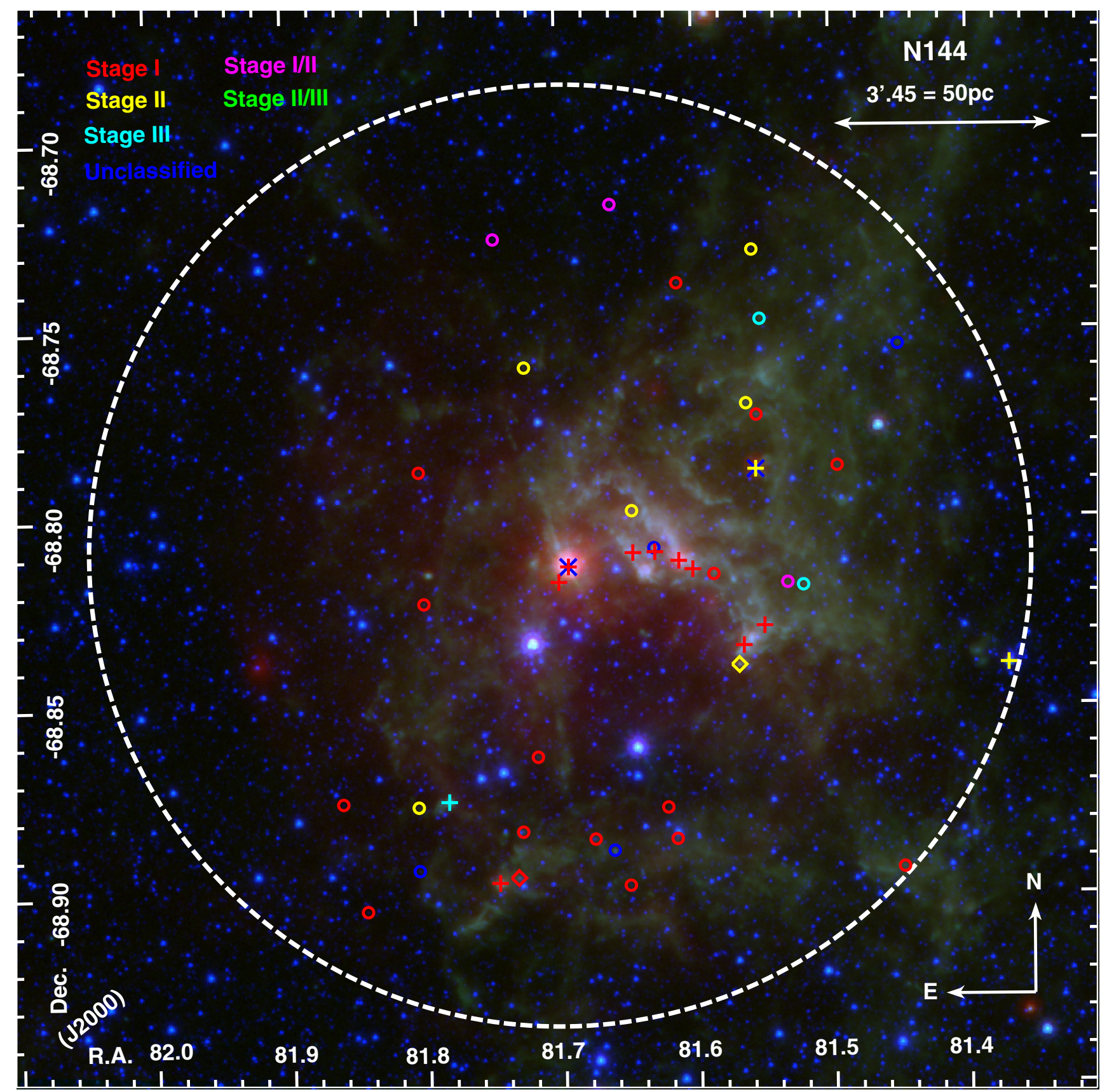

Fig. 11. Three-color image of N144 with YSO candidates marked on IRAC $3.6 \mu \mathrm{m}$ (blue) $+8.0 \mu \mathrm{m}$ (green) + MIPS $24 \mu \mathrm{m}$ (red). Candidate YSOs are marked as in Fig. 5.

Sixty-one previously identified candidates meet only one $\beta$ criterion: four from W08 and fifty-seven in Romita et al. (2010). This is to be expected, as Romita et al. (2010) require only one of their "faint" criteria to be met. These are listed as Set 10 in Table 2. Again, fitting is only performed for plotting purposes.

The remaining 176 candidates from other lists that we do not replicate are color-selected but do not meet our $\chi^{2}$ criteria for good SED fits. These include three sources in N120 and N160 which Seale et al. (2009) found to be unembedded sources (see Sects. 4.6 and 4.8). Another 34 of these colorselected candidates can be well-fit both as YSOs and by templates for galaxies, AGBs, and/or naked stars. We consider these fits ambiguous and note them in Table 3. Note that W08 and GC09 candidates were chosen via color-selection, and SEDs and images were visually inspected. Neither applied a quantitative SED fitting requirement, i.e. there were no $\chi^{2}$ requirements for matching YSO model SEDs.

Romita et al. (2010) identified and classified $\sim 100$ YSO candidates in N206, using the same photometry and YSO model fitter we use here. Our new examination reveals 73 well-fit sources, only 23 of which fall in the final Romita et al. (2010) catalog. We find that 57 of the Romita et al. (2010) sources fit only one of our $\beta$ selection criteria and zero $\alpha$ criteria; two more were not selected in any of our CMD cuts, accounting for most of the discrepancy in the number of identified candidates. Romita et al. (2010) used more complicated selection criteria of W08 with the addition of faint color-selections. They also examined the SED and multiple wavelength images of every source, allowing quality cuts to include higher values of $\chi^{2} / \mathrm{pt}$ 


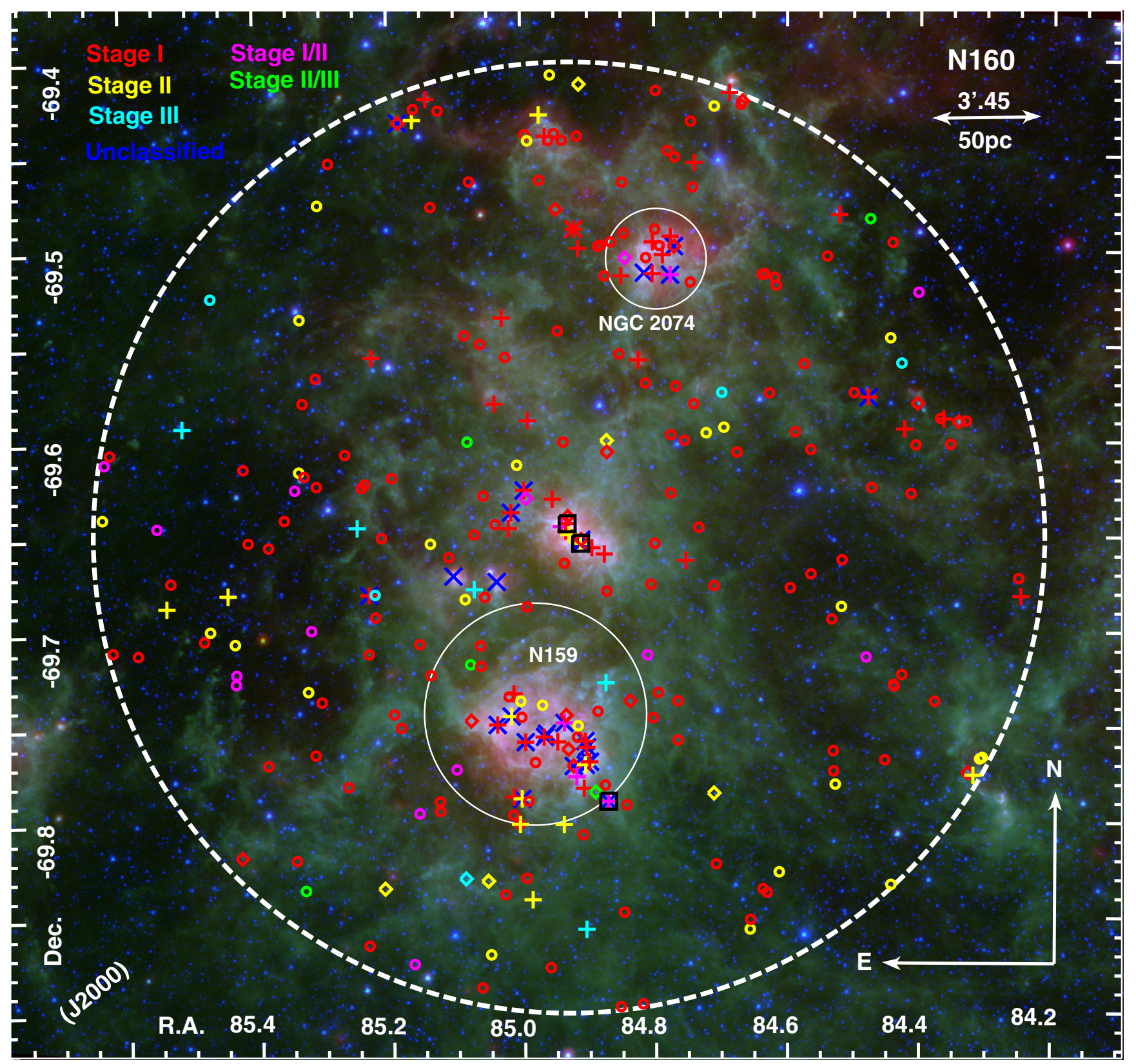

Fig. 12. Three-color images of N160 with YSO candidates marked. a) Full region in IRAC $3.6 \mu \mathrm{m}$ (blue) $+8.0 \mu \mathrm{m}$ (green) + MIPS $24 \mu \mathrm{m}$ (red). Candidate YSOs are marked as in Fig. 5. Crosses represent sources from W08, GC09, Chen et al. (2010), and Fleener et al. (2010). Large black squares are water masers from Ellingsen et al. (2010) as discussed in text. The three brightest and most active regions are, from north to south NGC 2074, NGC 2080, and N159.

and allowing the inclusion of objects meeting only one "faint" color-selection. Where appropriate, they added upper-limits to $24 \mu \mathrm{m}$ fluxes by hand, allowing more sources to be fit with higher accuracy. Fourteen candidates were removed from the Romita et al. (2010) list based on the double-peaked morphology of their SEDs suggesting multiple sources or "transition objects", with significant gaps between the central stellar object and the inner edge of the disk. None of these sources satisfy our new selection criteria; ten are selected by only one $\beta$-cut, and the four selected in Sets 4-9 do not fulfill our $\chi^{2}$ criteria.

\subsection{Mass functions and star formation rates}

We also use the derived masses to construct a mass function (MF; Fig. 14) and derive a star formation rate (SFR) for each region. In constructing the mass function histograms, we include only Stage I sources, to treat a population of roughly equivalent age. We know that we are incomplete at the low-mass end because of sensitivity and at the high-mass end due to saturation, crowding, and extension. We therefore fit the peak of the observed mass distribution where we expect approximate completeness and assume a standard two-part IMF (Kroupa 2001) from 0.08 to $50 M_{\odot}$, with $\xi \propto M_{\text {star }}^{-1.3}$ for $M_{\text {star }}<0.5 M_{\odot}$ and $\xi \propto M_{\text {star }}^{-2.3}$ for $M_{\text {star }}>0.5 M_{\odot}$. We report the (Stage I) integrated mass and SFRs for each region in Table 8.

We calculate SFR for each region using a "bottoms-up" approach. We divide the total integrated mass from the MF by two possible timescales for the Stage I formation, $1 \mathrm{Myr}$ and 0.2 Myr as adopted by W08 for Stage I sources in the LMC. For comparison, we also report SFRs using a top-down approach, 


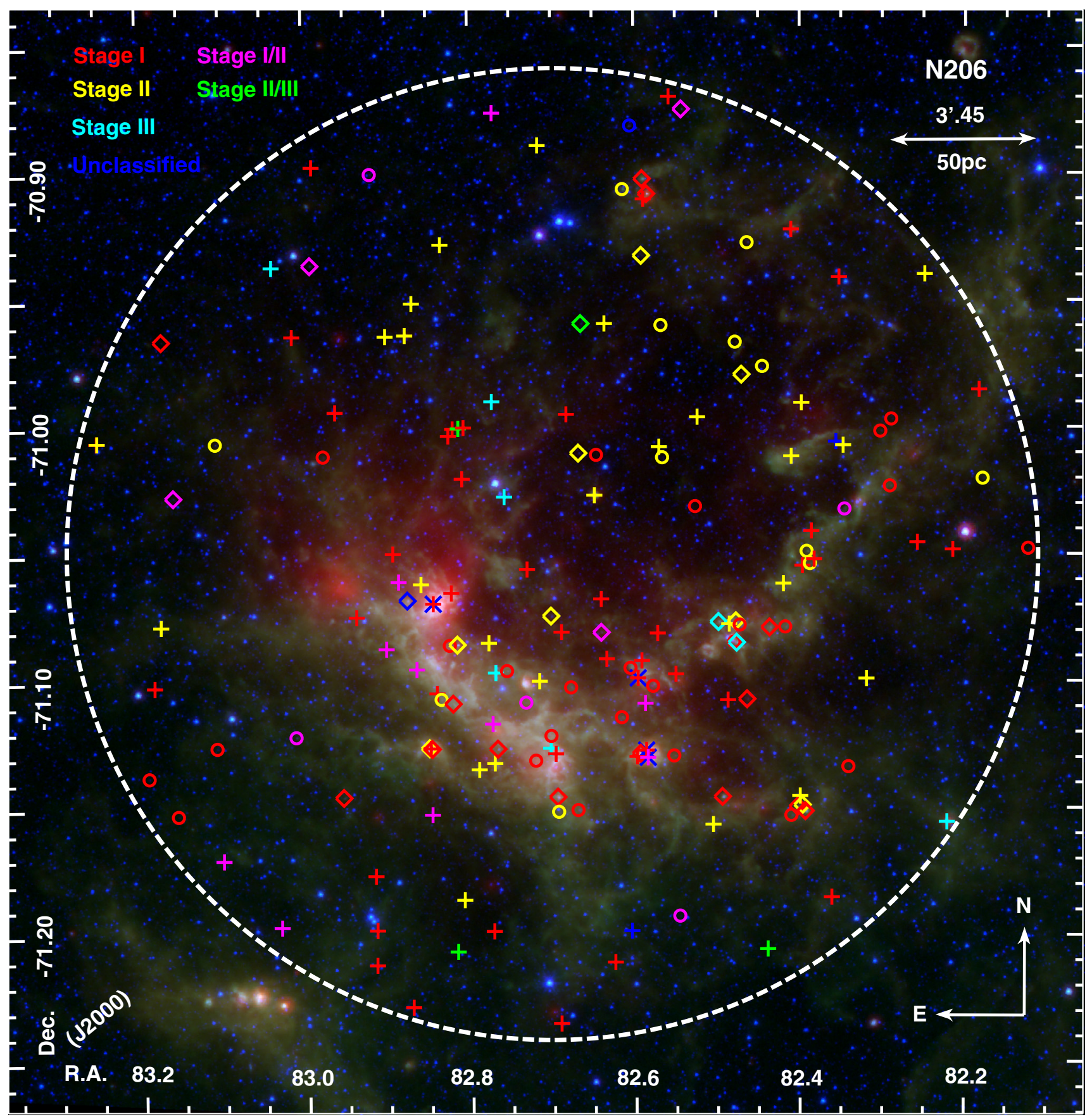

Fig. 13. Three-color image of N206 with YSO candidates marked on IRAC $3.6 \mu \mathrm{m}$ (blue) $+8.0 \mu \mathrm{m}$ (green) + MIPS $24 \mu \mathrm{m}$ (red). Candidate YSOs are marked as in Fig. 5. Squares are candidates from Romita et al. (2010).

in which the integrated $70 \mu \mathrm{m}$ flux is used as a proxy for the total infrared (as in Lawton et al. 2010). While this integrated $70 \mu \mathrm{m}$ flux is more likely to be contaminated by diffuse interstellar radiation heating the cold dust, these SFRs are calculated for the exact regions we examine here, known star-forming regions where we expect the IR to be dominated by forming stars. The Lawton et al. (2010) method rooted on Kennicutt (1998) and subsequent work, which is calibrated for regions of hundreds of parsecs to galaxy scales. We are at the lower end of the applicable scale. Our "bottoms-up" approach yields similar results to the top-down approach if we assume a star-formation timescale of 1 Myr. For N11, N44, and N144, the SFR per area calculated from our sources is within $\sim 5 \%$ the SFR from the Lawton et al. (2010) method. Our N105 value is $\sim 35 \%$ higher than that obtained from $70 \mu \mathrm{m}$, and our N206 value is $\sim 50 \%$ higher than the $70 \mu \mathrm{m}$ would suggest. N113 and N51 SFRs obtained from the bottoms-up method are a factor of 1.9 and 2.25 greater than the $70 \mu \mathrm{m}$ flux indicates. Regions N120 and N160 show bottoms-up SFRs significantly lower than the $70 \mu \mathrm{m}$ SFR, at $66 \%$ in $\mathrm{N} 120$ and less than 59\% in N160. Paired with the discrepancy between the unusually high percentage of Stage I candidates, this may indicate that SFR is increasing in N160.

\section{Summary and ending remarks}

Our new color-selection criteria are can be used in combination with SED fitting to select highly reliable candidates. We increase 


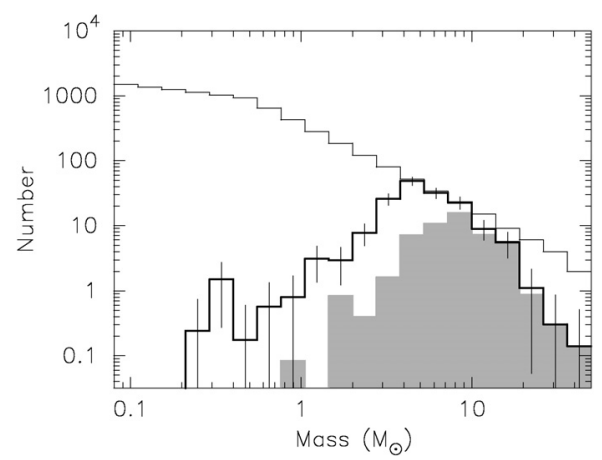

(a) N11

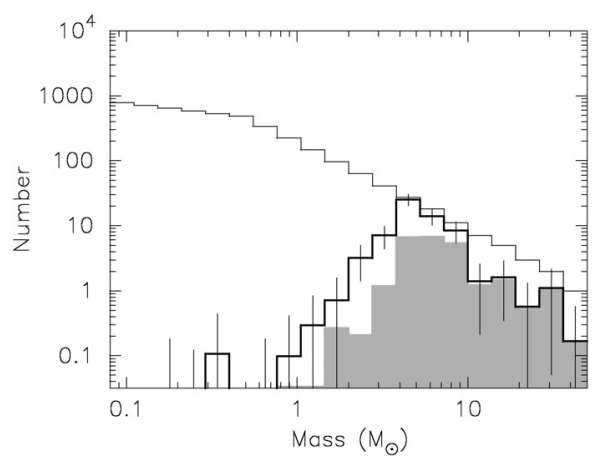

(d) N105

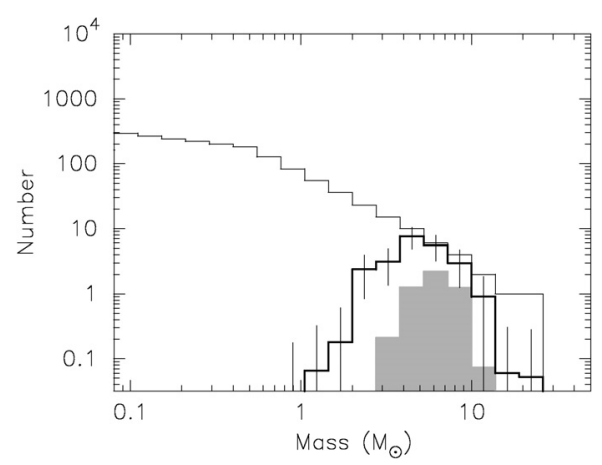

(g) N144

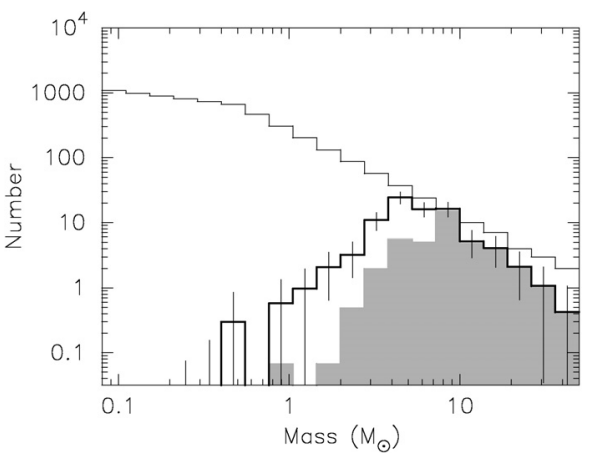

(b) N44

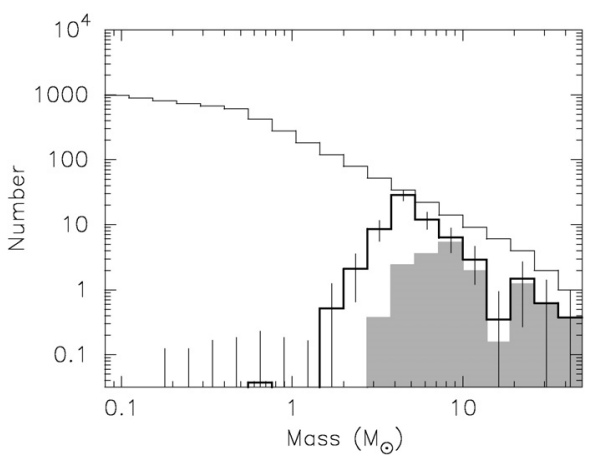

(e) N113

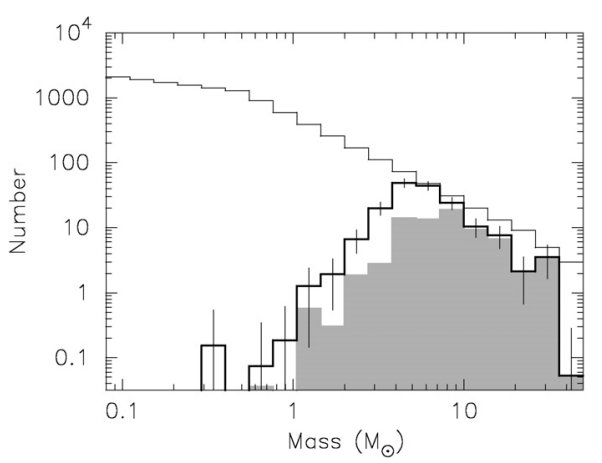

(h) N160

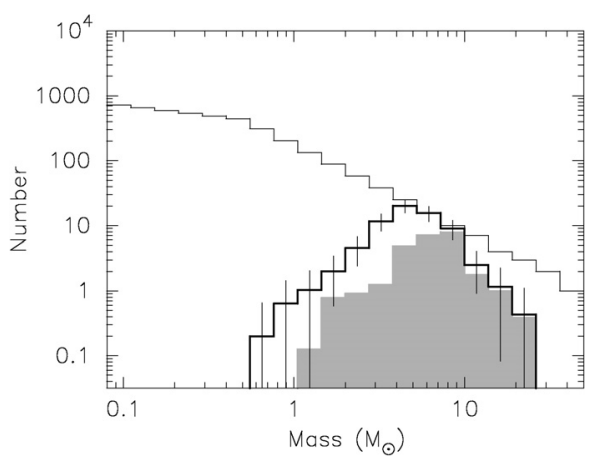

(c) N51

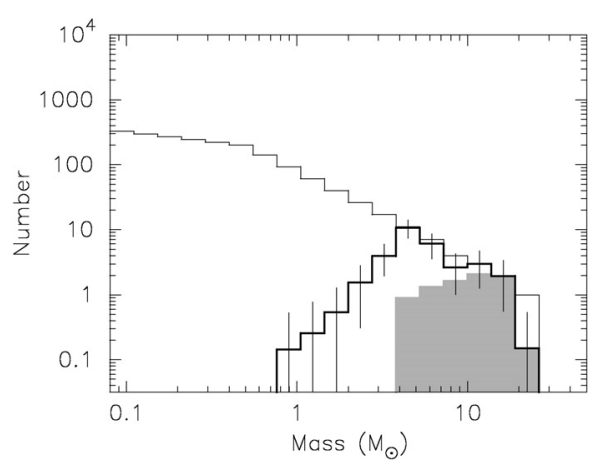

(f) $\mathrm{N} 120$

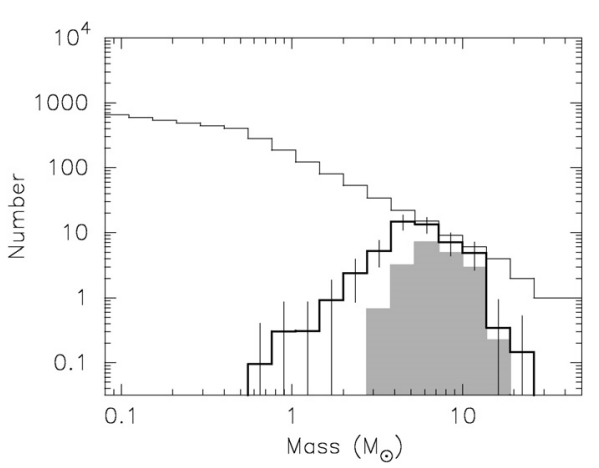

(i) $\mathrm{N} 206$

Fig. 14. Mass function of the YSO candidates in each cluster. The full well-fit lists are under the thick black line. Top lines show the Kroupa IMF. The shaded portion indicates $\alpha$-selected sources. a) N11: total mass of all 242 well-fit sources is $1391 \pm 26 M_{\odot}$ with mass from integrated Kroupa IMF $\sim 4905 M_{\odot}$. b) N44: mass of 139 well-fit sources: $900 \pm 25 M_{\odot}$; from integrated Kroupa IMF $\sim 3537 M_{\odot}$. c) N51: mass of 103 well-fit sources: $557 \pm 17 M_{\odot}$; from integrated Kroupa IMF $\sim 2339 M_{\odot}$. d) N105: mass of 91 well-fit sources: $526 \pm 14 M_{\odot}$; from integrated Kroupa IMF $\sim 2539 M_{\odot}$. e) N113: mass of 101 well-fit sources: $586 \pm 15 M_{\odot}$; from integrated Kroupa IMF $\sim 3157 M_{\odot}$. f) N120: mass of 51 well-fit sources: $291 \pm 10 M_{\odot}$; from integrated Kroupa IMF $\sim 998 M_{\odot}$. g) N144: mass of 31 well-fit sources: $163 \pm 8 M_{\odot}$; from integrated Kroupa IMF $\sim 879 M_{\odot}$. h) N160: mass of 214 well-fit sources: $1424 \pm 24 M_{\odot}$; from integrated Kroupa IMF $\sim 6841 M_{\odot}$. i) N206: mass of 73 well-fit sources: $431 \pm 12 M_{\odot}$; from integrated Kroupa IMF $\sim 2088 M_{\odot}$.

the total number of YSO candidates reported in these 9 starforming regions from 485 (in Whitney et al. 2008; Gruendl \& Chu 2009; Chen et al. 2009, 2010; Romita et al. 2010) to 1403, a factor of 3 increase. Without fitting SEDs, the color-selection includes $>99 \%$ of previous candidates. Our selections have the strength of using all four IRAC bands and MIPS $24 \mu \mathrm{m}$ without losing sources with one or two missing bands. In particular, our $\beta$ criteria allow the selection of lower mass YSO candidates in star-forming regions. Coupled with SED fitting, our full selection criteria largely avoids the selection of background galaxies and known non-YSOs.

We present new YSO candidate lists for each of the 9 star-forming regions covering a wide variety of physical conditions. As expected, our well-fit candidates are concentrated along dust ridges and near previously known YSOs. We take physical properties for each YSO candidate from the SED fits and derive SFRs for each region. These SFRs agree well with SFRs from the Lawton et al. (2010) $70 \mu \mathrm{m}$ TIR proxy for the SFR calculations of Kennicutt (1998) if we assume a $1 \mathrm{Myr}$ timescale for star formation. As with other studies, the majority $(\sim 56 \%)$ of our YSO candidates are classified as Stage I. Another $\sim 22 \%$ are classified as Stage II and $\sim 12 \%$ as Stage I/II, such that $\sim 90 \%$ of our identified YSO candidates have stages earlier than Stage II/III. This is largely a question of sensitivity at the wavelengths analyzed, as slightly older PMS sources, having lost most of their circumstellar matter are fainter in the infrared. 
Table 8. Star formation rates.

\begin{tabular}{|c|c|c|c|c|c|c|c|}
\hline Region & $\begin{array}{r}\mathrm{Fit}^{a} \\
(\#)\end{array}$ & $\begin{array}{r}\text { Mass }^{b} \\
\left(M_{\odot}\right)\end{array}$ & $\begin{array}{r}S F R_{1}{ }^{c} \\
\left(10^{-4}\right.\end{array}$ & $\begin{array}{c}S F R_{0.2}{ }^{d} \\
\left.M_{\odot} \mathrm{yr}^{-1}\right)\end{array}$ & $S F R_{1}{ }^{c, e}$ & \multicolumn{2}{|c|}{$\left(10^{-2} M_{\odot} \mathrm{yr}^{-1} \mathrm{kpc}^{-2}\right)$} \\
\hline N11 & 242 & 4905 & 49.0 & 245 & 1.8 & 8.8 & $1.7 \pm 0.1$ \\
\hline N44 & 139 & 3537 & 35.4 & 177 & 3.2 & 16.1 & $3.1 \pm 0.3$ \\
\hline N51 & 103 & 2339 & 23.4 & 117 & 2.7 & 13.4 & $1.2 \pm 0.1$ \\
\hline N105 & 91 & 2539 & 25.4 & 127 & 2.5 & 12.3 & $2.0 \pm 0.1$ \\
\hline N113 & 101 & 3157 & 31.6 & 158 & 4.0 & 19.8 & $2.1 \pm 0.1$ \\
\hline N120 & 51 & 998 & 10.0 & 50 & 1.2 & 6.2 & $1.9 \pm 0.1$ \\
\hline N144 & 31 & 879 & 8.8 & 44 & 2.4 & 11.9 & $2.5 \pm 0.2$ \\
\hline N160 & 214 & 6841 & 68.4 & 342 & 4.6 & 23.0 & $7.8 \pm 0.6$ \\
\hline N206 & 73 & 2088 & 20.9 & 104 & 2.4 & 12.0 & $1.6 \pm 0.1$ \\
\hline
\end{tabular}

Notes. Integrated masses and SFRs for each region. ${ }^{(a)}$ Number of Sources Fit. ${ }^{(b)}$ Mass from integrated Kroupa MF. ${ }^{(c)}$ SFR assuming a time scale of $1 \mathrm{Myr} .{ }^{(d)}$ SFR assuming a time scale of 0.20 Myr. ${ }^{(e)}$ SFR divided by cluster area covered. ${ }^{(f)}$ SFR derived from $70 \mu \mathrm{m}$ integrated flux (as in Lawton et al. 2010).

Acknowledgements. This work has been supported by grants and contracts from NASA NAG5-12595, NASA ADP-NNX11AG50G, and JPL-Spitzer contract \#1275598. The authors would like to thank Barb Whitney, Remy Indebetouw, Joana Oliveira, and Jonathan Seale for helpful discussion and Sean Points and the MCELS team for providing the beautiful $\mathrm{H} \alpha$ image.

\section{References}

Ambrocio-Cruz, P., Laval, A., Marcelin, M., Amram, P., \& Comeron, F. 1998, A\&A, 339, 173

Barbá, R. H., Rubio, M., Roth, M. R., \& García, J. 2003, AJ, 125, 1940

Bernasconi, P. A., \& Maeder, A. 1996, A\&A, 307, 829

Bica, E., Claria, J. J., Dottori, H., Santos, Jr., J. F. C., \& Piatti, A. E. 1996, ApJS, 102, 57

Breysacher, J., Azzopardi, M., \& Testor, G. 1999, A\&AS, 137, 117

Brooks, K. J., \& Whiteoak, J. B. 1997, MNRAS, 291, 395

Carlson, L. R., Sewiło, M., Meixner, M., et al. 2011, ApJ, 730, 78

Chen, C., Chu, Y., Gruendl, R. A., Gordon, K. D., \& Heitsch, F. 2009, ApJ, 695, 511

Chen, C., Indebetouw, R., Chu, Y., et al. 2010, ApJ, 721, 1206

Chu, Y., Mac Low, M., Garcia-Segura, G., Wakker, B., \& Kennicutt, Jr., R. C. 1993, ApJ, 414, 213

Chu, Y., Gruendl, R. A., Chen, C., et al. 2005, ApJ, 634, L189

Danziger, I. J., \& Dennefeld, M. 1974, A\&A, 36, 149

Dufour, R. J., Shields, G. A., \& Talbot, Jr., R. J. 1982, ApJ, 252, 461

Dunne, B. C., Points, S. D., \& Chu, Y. 2001, ApJS, 136, 119

Ellingsen, S. P., Whiteoak, J. B., Norris, R. P., Caswell, J. L., \& Vaile, R. A. 1994, MNRAS, 269, 1019

Ellingsen, S. P., Breen, S. L., Caswell, J. L., Quinn, L. J., \& Fuller, G. A. 2010, MNRAS, 404, 779

Epchtein, N., Braz, M. A., \& Sevre, F. 1984, A\&A, 140, 67

Fariña, C., Bosch, G. L., Morrell, N. I., Barbá, R. H., \& Walborn, N. R. 2009, AJ, 138, 510

Fazio, G. G., Hora, J. L., Allen, L. E., et al. 2004, ApJS, 154, 10

Fleener, C. E., Payne, J. T., Chu, Y., Chen, C., \& Gruendl, R. A. 2010, AJ, 139, 158

Green, J. A., Caswell, J. L., Fuller, G. A., et al. 2008, MNRAS, 385, 948

Gruendl, R. A., \& Chu, Y. 2009, ApJS, 184, 172

Hatano, H., Kadowaki, R., Nakajima, Y., et al. 2006, AJ, 132, 2653

Henize, K. G. 1956, ApJS, 2, 315

Heydari-Malayeri, M., Royer, P., Rauw, G., \& Walborn, N. R. 2000, A\&A, 361, 877

Heydari-Malayeri, M., Charmandaris, V., Deharveng, L., et al. 2001, A\&A, 372, 527

Hora, J. L., Cohen, M., Ellis, R. G., et al. 2008, AJ, 135, 726

Indebetouw, R., Johnson, K. E., \& Conti, P. 2004, AJ, 128, 2206

Israel, F. P., Maloney, P. R., Geis, N., et al. 1996, ApJ, 465, 738

Johansson, L. E. B., Greve, A., Booth, R. S., et al. 1998, A\&A, 331, 857

Kato, D., Nagashima, C., Nagayama, T., et al. 2007, PASJ, 59, 615

Kennicutt, Jr., R. C. 1998, ARA\&A, 36, 189

Kim, S., Chu, Y., Staveley-Smith, L., \& Smith, R. C. 1998, ApJ, 503, 729

Kroupa, P. 2001, MNRAS, 322, 231

Lada, C. J. 1987, in Star Forming Regions, ed. M. Peimbert, \& J. Jugaku, IAU Symp., 115, 1

Laval, A., Rosado, M., Boulesteix, J., et al. 1992, A\&A, 253, 213
Lawton, B., Gordon, K. D., Babler, B., et al. 2010, ApJ, 716, 453

Lazendic, J. S., Whiteoak, J. B., Klamer, I., Harbison, P. D., \& Kuiper, T. B. H. 2002, MNRAS, 331, 969

Lortet, M., \& Testor, G. 1988, A\&A, 194, 11

Lucke, P. B., \& Hodge, P. W. 1970, AJ, 75, 171

Massey, P., Waterhouse, E., \& DeGioia-Eastwood, K. 2000, AJ, 119, 2214

Meaburn, J., \& Laspias, V. N. 1991, A\&A, 245, 635

Meaburn, J., \& Terrett, D. L. 1980, A\&A, 89, 126

Meixner, M., Gordon, K. D., Indebetouw, R., et al. 2006, AJ, 132, 2268

Moffat, A. F. J., Niemela, V. S., \& Marraco, H. G. 1990, ApJ, 348, 232

Mokiem, M. R., de Koter, A., Evans, C. J., et al. 2007, A\&A, 465, 1003

Nakajima, Y. 2005, J. Korean Astron. Soc., 38, 173

Nakajima, Y., Kato, D., Nagata, T., et al. 2005, AJ, 129, 776

Nazé, Y., Antokhin, I. I., Rauw, G., et al. 2004, A\&A, 418, 841

Oey, M. S., \& Massey, P. 1995, ApJ, 452, 210

Oey, M. S., \& Smedley, S. A. 1998, AJ, 116, 1263

Oliveira, J. M., van Loon, J. T., Stanimirović, S., \& Zijlstra, A. A. 2006, MNRAS, 372, 1509

Panagia, N. 2005, in Cosmic Explosions, On the 10th Anniversary of SN1993J, ed. J.-M. Marcaide, \& K. W. Weiler, IAU Colloq., 192, 585

Pei, Y. C., Fall, S. M., \& Hauser, M. G. 1999, ApJ, 522, 604

Reid, W. A., \& Parker, Q. A. 2006, MNRAS, 373, 521

Rieke, G. H., Young, E. T., Engelbracht, C. W., et al. 2004, ApJS, 154, 25

Robitaille, T. P., Whitney, B. A., Indebetouw, R., Wood, K., \& Denzmore, P. 2006, ApJS, 167, 256

Robitaille, T. P., Whitney, B. A., Indebetouw, R., \& Wood, K. 2007, ApJS, 169, 328

Romita, K. A., Carlson, L. R., Sewilo, M., et al. 2010, ApJ, 721, 357

Rosado, M., Laval, A., Le Coarer, E., et al. 1996, A\&A, 308, 588

Schaefer, B. E. 2008, AJ, 135, 112

Seale, J. P., Looney, L. W., Chu, Y.-H., et al. 2009, ApJ, 699, 150

Sewiło, M., Indebetouw, R., Carlson, L. R., et al. 2010, A\&A, 518, L73

Shimonishi, T., Onaka, T., Kato, D., et al. 2008, ApJ, 686, L99

Sinclair, M. W., Carrad, G. J., Caswell, J. L., Norris, R. P., \& Whiteoak, J. B. 1992, MNRAS, 256, 33

Skrutskie, M. F., Cutri, R. M., Stiening, R., et al. 2006, AJ, 131, 1163

Smith, R. C., \& MCELS Team 1998, PASA, 15, 163

Smith, R. C., Points, S. D., Chu, Y.-H., et al. 2005, in BAAS, 37, AAS Meeting Abstracts, 1200

Srinivasan, S., Meixner, M., Leitherer, C., et al. 2009, AJ, 137, 4810

Stasińska, G., Testor, G., \& Heydari-Malayeri, M. 1986, A\&A, 170, L4

Vallenari, A., Chiosi, E., \& Sordo, R. 2010, A\&A, 511, A79

van der Marel, R. P., \& Cioni, M. 2001, AJ, 122, 1807

Walborn, N. R., \& Parker, J. W. 1992, ApJ, 399, L87

Walborn, N. R., Drissen, L., Parker, J. W., et al. 1999, AJ, 118, 1684

Weinberg, M. D., \& Nikolaev, S. 2001, ApJ, 548, 712

Westerlund, B. E. 1997, The Magellanic Clouds (Cambridge University Press)

Whiteoak, J. B., \& Gardner, F. F. 1986, MNRAS, 222, 513

Whitney, B. A., Wood, K., Bjorkman, J. E., \& Wolff, M. J. 2003a, ApJ, 591, 1049

Whitney, B. A., Sewiło, M., Indebetouw, R., et al. 2008, AJ, 136, 18

Will, J., Bomans, D. J., \& Dieball, A. 1997, A\&AS, 123, 455

Wong, T., Whiteoak, J. B., Ott, J., Chin, Y., \& Cunningham, M. R. 2006, ApJ, 649,224

Woods, P. M., Oliveira, J. M., Kemper, F., et al. 2011, MNRAS, 411, 1597

Zaritsky, D., Harris, J., \& Thompson, I. 1997, AJ, 114, 1002 